Geometry $\mathcal{E}_{\mathcal{G}} \mathcal{T}_{\text {opology }}$

Volume 5 (2001) 75-108

Published: 10 February 2001

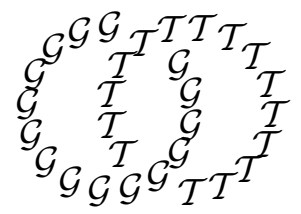

\title{
Calculus of clovers and finite type invariants of 3-manifolds
}

\author{
Stavros Garoufalidis \\ MikHail Goussarov \\ Michael PolyaK \\ SG: School of Mathematics, Georgia Institute of Technology \\ Atlanta, GA 30332-0160 USA \\ MP: School of Mathematics, Tel-Aviv University \\ 69978 Tel-Aviv, Israel \\ Email: stavros@math.gatech.edu and polyak@math.tau.ac.il
}

\begin{abstract}
A clover is a framed trivalent graph with some additional structure, embedded in a 3-manifold. We define surgery on clovers, generalizing surgery on Y-graphs used earlier by the second author to define a new theory of finite-type invariants of 3-manifolds. We give a systematic exposition of a topological calculus of clovers and use it to deduce some important results about the corresponding theory of finite type invariants. In particular, we give a description of the weight systems in terms of uni-trivalent graphs modulo the AS and IHX relations, reminiscent of the similar results for links. We then compare several definitions of finite type invariants of homology spheres (based on surgery on Y-graphs, blinks, algebraically split links, and boundary links) and prove in a self-contained way their equivalence.
\end{abstract}

AMS Classification numbers Primary: 57N10, 57M27

Secondary: 57M25

Keywords: 3-manifolds, Y-graphs, finite type invariants, clovers

Proposed: Robion Kirby

Seconded: Joan Birman, Cameron Gordon
Received: 19 October 2000

Accepted: 28 January 2001

(C) Geometry 8 Topology Publications 


\section{Introduction}

\section{$1.1 \quad$ Finite type invariants}

Polynomials play a fundamental role in mathematics. Here is one of the possible definitions of a polynomial using a discrete version of the $n$-th derivative. Let $V$ be a vector space over a field $k$. For $\left(x_{1}, \ldots, x_{n}\right) \in V^{n}$ and $\sigma \in\{0,1\}^{n}$, denote $x_{\sigma}=\sum_{i: \sigma_{i}=1} x_{i}$ and $|\sigma|=\sum_{i} \sigma_{i}$. A function $f: V \rightarrow k$ is a polynomial of degree less than $n$, iff $\sum_{\sigma}(-1)^{|\sigma|} f\left(x_{\sigma}\right)=0$ for any $\left(x_{1}, \ldots, x_{n}\right) \in V^{n}$. This definition has a significant advantage over the standard definition of a polynomial, since it can be easily considered in a more general setup. In particular, $V$ and $k$ may be just abelian groups.

Finite type invariants play the role of polynomial functions in topology. In this setting, however, the situation is somewhat more tricky, and involves a choice of an appropriate analog of both the zero element 0 and the addition operation + in $V$. In the last decade, this general idea was successfully used in knot theory under the name of Vassiliev invariants. This paper is devoted exclusively to a study of a theory of finite type invariants of 3-manifolds, initiated by the second author.

The present paper was started in September 1998 in an attempt to present unpublished results by the second author. In June 1999, when this paper was still in its initial stage, the second author passed away in a tragic accident. As a result, many details and proofs were lost. We tried our best to reconstruct them and to present our results in his style and spirit.

The authors were partially supported by BSF grant $97-00398$ and by NSF grant DMS-98-00703.

\subsection{Finite type invariants of 3-manifolds}

Several authors developed different theories of finite type invariants of integer homology spheres. All these approaches are based on the cut-and-paste technique along different types of handlebodies. Historically the first, the theory of T Ohtsuki [13] is based on surgery on algebraically split links and is by now relatively well understood. The first author [2] proposed then a theory based on surgery on boundary links, which remained relatively undeveloped. Subsequently, the first author and J Levine [3] considered theories based on surgery on blinks, and on gluings along surfaces using elements of the Torelli group. All these theories turn out to be equivalent (up to a 2-torsion), see Section 1.8. 
The second author initiated in [5] a new theory of finite type invariants for arbitrary 3-manifolds, based on surgery along $\mathrm{Y}$-graphs (see $[4,5]$ ). The latter theory has some important advantages. Firstly, it allows a unified treatment of links, graphs and 3-manifolds (possibly with boundary or a Spin-structure). It is by now relatively well understood for rational homology spheres. Secondly, other theories mentioned above have a technical drawback: the corresponding classes of surgery links are not preserved under handle slides. Finally, this theory comes equipped with a powerful topological calculus (introduced in [4]), that is well-suited for a study of 3-manifolds and for explicit computations. This calculus is similar to Kirby's calculus of framed links, but instead of cutting, twisting and regluing solid tori, in the clover calculus one performs similar operations with solid handlebodies of higher genus. The role of framed links is played here by clovers, which are framed trivalent graphs with some additional structure.

The main goals of the paper are:

- To give an exposition of the calculus of clovers (see Sections 2-3), and to describe the graded spaces of the theory in terms of uni-trivalent graphs (see Sections 1.8 and 4.5).

- To show, as an application, in an elementary and self-contained way, that all the above mentioned theories of finite type invariants of integer homology spheres, properly indexed, coincide over $\mathbb{Z}[1 / 2]$ (see Section 1.8).

Independently and being uninformed about the results of the second author, K Habiro developed a theory of claspers [6] and used it to study finite type invariants of links. He also announced in [6, Section 8.4] that his theory can be extended to the study of finite type invariants of 3-manifolds and gave a brief outline of this extension. It is based on allowable graph claspers, which differ from the type of claspers considered in the rest of Habiro's paper. In particular, the basic claspers, which were Habiro's main tool in the theory for links, are not allowed in this setting. In general it is plausible that invariants of links can be extended to invariants of 3-manifolds. In the case in hand, however, this extension is rather nontrivial and involves new ideas presented here.

The initial data, the way to describe the basic objects, and the graphical calculus in the clasper and clover theories are somewhat different. However, while different in some aspects, these two theories essentially coincide, with surgery on allowable graph claspers being equivalent to surgery on clovers. This independent discovery of almost the same theory by two non-interacting researchers 
looks rather promising, ruling out an element of arbitrariness. Some results announced by K Habiro [6, Section 8.4] seem to overlap with those discussed in Sections 2-4 below. Unfortunately, it seems that presently there is no available manuscript with the exact statements and proofs of Habiro's results for 3-manifolds. We apologize in advance for any possible overlaps. It should be also mentioned that $\mathrm{T}$ Cochran and $\mathrm{P}$ Melvin [1] proposed an extension of Ohtsuki's theory to arbitrary 3-manifolds. Their theory, unlike the one considered in this paper, preserves triple cup-products, and the relation between these theories is more complicated.

\section{$1.3 \quad \mathrm{Y}$-graphs}

Throughout this paper by a manifold we mean a smooth oriented compact connected 3-manifold.

We recall some definitions from [5]. The graph $\Gamma \subset \mathbb{R}^{3}$ shown in Figure 1a is called a standard $Y$-graph. The edges of $\Gamma$ are framed with a vector field normal to the plane of the picture. A framed graph $G$ in a $3-$ manifold $M$ is called a $Y$-graph, if it is the image of $\Gamma$ under a smooth embedding $\phi_{G}: N \rightarrow M$ of a neighborhood $N$ of $\Gamma$. The embedding $\phi_{G}$ can be recovered from $G$ up to isotopy. Let $L$ be the six component link in $N$ shown in Figure 1b. All components of $L$ are 0-framed. Surgery on $M$ along the framed link $\phi_{G}(L)$ is called a surgery along $G$, or a $Y$-surgery. A Y-surgery can be realized by cutting a solid genus 3 handlebody $\phi_{G}(N)$ and regluing it in another way (see [10]). The resulting manifold is defined up to a diffeomorphism, which is the identity outside of a small neighborhood of the Y-graph. Denote it by $M_{G}$.
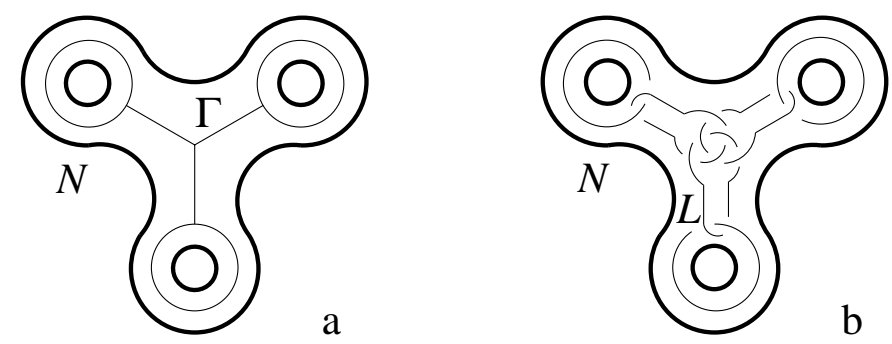

Figure 1: Y-graph and the corresponding surgery link

An equivalent surgery, under the name of Borromean surgery was considered by S Matveev. As shown in [10], two manifolds can be connected by a sequence of Borromean surgeries, if and only if they have the same homology and the linking 
pairing in Tors $H_{1}$. In particular, $M$ can be obtained from $S^{3}$ by $\mathrm{Y}$-surgeries, iff $M$ is an integer homology sphere ( $\mathbb{Z} H S$ in short).

A $Y$-link $G$ in a manifold $M$ is a collection of disjoint $Y$-graphs in $M$. By surgery on $G$ we mean surgery on each Y-graph in $G$. Pushing out the Ygraphs of the following surgeries from the previously reglued handlebodies, we can present a sequence of $\mathrm{Y}$-surgeries by surgery on a $\mathrm{Y}$-link.

\subsection{Colored $\mathrm{Y}$-links and the filtration $\mathcal{F}_{n}^{Y}(M)$}

An $n$-coloring of a Y-link $G$ is its splitting into $n$ disjoint subcollections $G_{1}$, $G_{2}, \ldots, G_{n}$ of Y-graphs. The coloring is simple, if each subcollection $G_{i}$ consists of a single $\mathrm{Y}$-graph.

For an $n$-tuple $\sigma=\left\{\sigma_{1}, \ldots, \sigma_{n}\right\} \in\{0,1\}^{n}$, let $|\sigma|=\sum_{i} \sigma_{i}$ be the number of ones in $\sigma$ and put $G(\sigma)=\bigcup_{i: \sigma_{i}=1} G_{i}$. In an abelian group $\mathcal{F}$ generated by all 3-manifolds, put

$$
[M, G]=\sum_{\sigma}(-1)^{|\sigma|} M_{G(\sigma)}
$$

for a colored Y-link $G$ in $M$.

Let $\mathcal{F}_{n}^{Y}(M)$ be the subgroup of $\mathcal{F}$, generated by $[M, G]$ for all $\mathrm{Y}$-links $G$, colored in at least $n$ colors. This defines a decreasing filtration $\mathcal{F}^{Y}(M)=$ $\mathcal{F}_{0}^{Y}(M) \supset \mathcal{F}_{1}^{Y}(M) \supset \mathcal{F}_{2}^{Y}(M) \supset \ldots$

Given a $Y$-link $G$ in $M$ without a specified coloring, by $[M, G]$ we mean the above alternating sum for a simple coloring of $G$.

Lemma 1.1 The subgroup $\mathcal{F}_{n}^{Y}(M)$ is generated by $[M, G]$ for all simply colored $Y$-links $G$ with at least $n$ components.

Proof Let $G$ be an $n$-colored Y-link with $m$ components. We call $d=n-m$ the defect of the coloring. If $d=0$, the coloring is simple. Suppose $d>0$; then there are at least two components of the same color $j$. Therefore, the $j$-colored Y-sublink of $G$ can be split into two disjoint non-empty parts $G^{\prime}$ and $G^{\prime \prime}$. It is easy to see that

$$
[M, G]=\left[M, G \backslash G^{\prime}\right]+\left[M, G \backslash G^{\prime \prime}\right]-[M, \widetilde{G}],
$$

where $\widetilde{G}$ is obtained from $G$ by recoloring all components of $G^{\prime \prime}$ in a new, $(n+1)$-th, color. The defect of the coloring for each of $G \backslash G^{\prime}, G \backslash G^{\prime \prime}$ and $\widetilde{G}$ is less than $d$. The statement follows by the induction on $d$.

Thus, without a loss of generality, one can assume that the colorings in the definition of $\mathcal{F}_{n}^{Y}(M)$ are simple. 


\subsection{The filtration $\mathcal{F}_{n}^{Y}$}

Further on we will be mainly interested in $M$ being a $\mathbb{Z}$ HS. Denote $\mathcal{F}_{n}^{Y}\left(S^{3}\right)$ by $\mathcal{F}_{n}^{Y}$.

Lemma 1.2 Let $G$ be an $n$-colored $Y$-link in a $\mathbb{Z}$ HS $M$. Then $[M, G] \in \mathcal{F}_{n}^{Y}$.

Proof It suffices to prove, that there exist two Y-links $G^{\prime}$ and $G^{\prime \prime}$ in $S^{3}$, each colored in at least $n$ colors, such that $[M, G]=\left[S^{3}, G^{\prime \prime}\right]-\left[S^{3}, G^{\prime}\right]$.

Since $M$ is a $\mathbb{Z}$ HS, it may be obtained from $S^{3}$ by Y-surgery on a Y-link $G$ in $S^{3}$. Let $G^{\prime}$ be an $n$-colored Y-link in $S^{3} \backslash G$, such that its image under Ysurgery on $G$ is isotopic to $G$. Let $G^{\prime \prime}$ be the $(n+1)$-colored Y-link, obtained from $G^{\prime}$ by an addition of $G$, with all components of $G$ colored in a new color. Then $\left[S^{3}, G^{\prime} \cup G\right]=\left[S_{G}^{3}, G\right]+\left[S^{3}, G^{\prime}\right]$ and hence $\left[S_{G}^{3}, G\right]=\left[S^{3}, G^{\prime \prime}\right]-\left[S^{3}, G^{\prime}\right]$.

\subsection{The filtrations $\mathcal{F}_{n}^{a s}$ and $\mathcal{F}_{n}^{b}$}

A link $L$ in a $\mathbb{Z H S}$ is algebraically split if all pairwise linking numbers of its components vanish. Such a link is boundary if all its components bound nonintersecting surfaces. A framing of $L$ is unimodular if the self-linking of each component is \pm 1 . Surgery on (any sublink of) a unimodular algebraically split link gives again a $\mathbb{Z}$ HS. Using these classes of links, T Ohtsuki [13] and the first author [2] introduced two different filtrations on a vector space generated by all $\mathbb{Z}$ HS. Below we describe the corresponding filtrations on the free abelian group generated by all $\mathbb{Z} \mathrm{HS}$.

For a framed link $L$ in $M$, denote by $M_{L}$ the result of surgery of $M$ along $L$. In $\mathcal{F}$, let

$$
[M, L]=\sum_{L^{\prime} \subset L}(-1)^{\left|L^{\prime}\right|} M_{L^{\prime}}
$$

where $\left|L^{\prime}\right|$ is the number of components of a sublink $L^{\prime}$. Let $\mathcal{F}_{n}^{\text {as }}$ (respectively $\mathcal{F}_{n}^{b}$ ) be the subgroup of $\mathcal{F}$, generated by $[M, L]$ for all algebraically split (respectively boundary) unimodular links $L$ in $\mathbb{Z}$ HS $M$ with at least $n$ components. As shown in [13] and [2], in the definitions of these filtrations it suffices to consider only $M=S^{3}$. 


\subsection{The filtration $\mathcal{F}_{n}^{b l}$}

In [3], J Levine and the first author considered another filtration, based on a notion of a blink. A blink is a framed link $B$ with a given splitting of the set of its components into pairs $\left(B_{1}^{-}, B_{1}^{+}\right), \ldots,\left(B_{n}^{-}, B_{n}^{+}\right)$, such that:

- each pair $\left(B_{i}^{-}, B_{i}^{+}\right)$bounds an orientable surface $\Sigma_{i}$, so that $\Sigma_{i} \cap \Sigma_{j}=\emptyset$ for $i \neq j$;

- the surface $\Sigma_{i}$ induces a preferred framing of $B_{i}^{-}$and $B_{i}^{+}$; this framing should differ by \pm 1 from the given framing of $B_{i}^{ \pm}$.

A sub-blink $B^{\prime}$ of $B$ is obtained from $B$ by a removal of some pairs $\left(B_{i}^{-}, B_{i}^{+}\right)$. In $\mathcal{F}$, put

$$
[M, B]=\sum_{B^{\prime} \subset B}(-1)^{\left|B^{\prime}\right|} M_{B^{\prime}},
$$

where $\left|B^{\prime}\right|$ is the number of pairs in a sub-blink $B^{\prime}$. Let $\mathcal{F}_{n}^{b l}(M)$ be the subgroup of $\mathcal{F}$, generated by $[M, L]$ for all blinks $L$ in a manifold $M$ with at least $n$ pairs of components.

In the same paper [3] several other filtrations were introduced, using different subgroups of the mapping class group. Each of them was shown to be equivalent to one of the above filtrations.

\subsection{The main results}

We describe a topological calculus of surgery on Y-links, developed by the second author. Using this calculus, we describe the structure of the graded groups $\mathcal{F}_{n}^{Y} / \mathcal{F}_{n+1}^{Y} \otimes \mathbb{Z}[1 / 2]$, where $\mathbb{Z}[1 / 2]=\left\{n / 2^{m} \mid n, m \in \mathbb{Z}\right\}$ is the group of binary rational numbers.

We also compare different filtrations:

- $\mathcal{F}_{n}^{Y}(M)=\mathcal{F}_{n}^{b l}(M)$;

- $\mathcal{F}_{2 n}^{Y} \subset \mathcal{F}_{3 n}^{a s}$ and $\mathcal{F}_{2 n}^{Y} \subset \mathcal{F}_{n}^{b}$;

- $\mathcal{F}_{2 n-1}^{Y} \otimes \mathbb{Z}[1 / 2]=\mathcal{F}_{2 n}^{Y} \otimes \mathbb{Z}[1 / 2]$;

- $\mathcal{F}_{2 n}^{Y} \otimes \mathbb{Z}[1 / 2]=\mathcal{F}_{3 n}^{a s} \otimes \mathbb{Z}[1 / 2]$ and $\mathcal{F}_{2 n}^{Y} \otimes \mathbb{Z}[1 / 2]=\mathcal{F}_{n}^{b} \otimes \mathbb{Z}[1 / 2]$.

Let $F$ be a free abelian group on a set $S$ of generators, equipped with a decreasing filtration $F=F_{0} \supset F_{1} \supset \ldots$ Given an abelian group $A$, a function $S \rightarrow A$ is called a finite type invariant of degree at most $d$, if its extension to a homomorphism $F \rightarrow A$ vanishes on $F_{d+1}$. Each filtration $\mathcal{F}_{n}^{a s}, \mathcal{F}_{n}^{b}, \mathcal{F}_{n}^{b l}$ 
and $\mathcal{F}_{n}^{Y}$ defines a notion of a finite type invariant on the set of all $\mathbb{Z}$ HS. The above comparison of filtrations implies the following theorem announced in $[5$, Theorem 1]. If 2 is invertible in $A$, these definitions are equivalent, with the following relation of degrees:

$$
2 d^{a s}=6 d^{b}=3 d^{b l}=3 d^{Y} .
$$

In [3], weaker results $\mathcal{F}_{n}^{b} \otimes \mathbb{Q} \subset \mathcal{F}_{2 n}^{b l} \otimes \mathbb{Q}=\mathcal{F}_{3 n}^{a s} \otimes \mathbb{Q}$ were obtained by using different methods that involved the study of the mapping class group and several of its subgroups (building on the results of D Johnson $[7,8]$ ).

\section{$2 \quad$ Surgery on clovers}

In this section we introduce a generalization of Y-links, which we call clovers. They turn out to be quite useful from a technical point of view, and are closely related to uni-trivalent graphs appearing in the study of the graded quotients $\mathcal{F}_{n}^{Y} / \mathcal{F}_{n+1}^{Y}$. Similar objects were called allowable graph claspers $^{1}$ by K Habiro [6]. We start by recalling some basic facts about surgery (see [9]).

\section{$2.1 \quad$ Surgery on links}

We will call framed links $L$ and $L^{\prime}$ in a manifold $M$ surgery equivalent, and denote $L \sim L^{\prime}$, if $M_{L}$ is diffeomorphic to $M_{L^{\prime}}$. Recall (see [9]), that links $L$ and $L^{\prime}$ in $S^{3}$ are surgery equivalent, iff one can pass from $L$ to $L^{\prime}$ by a sequence of Kirby moves $K_{1}$ and $K_{2}$ :

$K_{1}$ : Add to $L$ a small \pm 1 framed unknot, unlinked with the other components of $L$.

$K_{2}$ : Add $L_{0}$ to $L_{1}$ by replacing $L_{1}$ with $L_{1} \#_{b} \widetilde{L_{0}}$, where $\#_{b}$ is a band connected sum and $\widetilde{L_{0}}$ is a push-off of $L_{0}$ along the framing.

It is convenient to introduce three additional moves $K_{3}-K_{5}$, which can be expressed via $K_{1}^{ \pm 1}$ and $K_{2}$ (see [9] for $K_{4}, K_{5}$, and [11] for $K_{3}$ ):

$K_{3}$ : Let $L_{0}$ be a closed, 0 -framed component of $L$ bounding an embedded disk $D$, which intersects $L \backslash L_{0}$ in exactly two points, belonging to different components $L_{1}$ and $L_{2}$ of $L$. Replace $L_{0} \cup L_{1} \cup L_{2}$ by $L_{1} \#_{b} L_{2}$, where the band $b$ intersects $D$ along the middle line of $b$.

\footnotetext{
${ }^{1}$ See http://www.dictionary.com for some unexpected meanings of the word clasper.
} 
$K_{4}$ : Delete a \pm 1 -framed unknot, at the expense of the full left- or right-hand twist on the strings linked with it.

$K_{5}$ : Let $L_{0}$ be a closed, 0 -framed component of $L$ which bounds an embedded disk $D$. Suppose that $D \cap\left(L \backslash L_{0}\right)$ consists of exactly one point lying on some component $L_{1} \subset L$.

Delete $L_{0}$ and $L_{1}$ from $L$.

The moves $K_{2}-K_{5}$ are shown in Figure 2. Here, and throughout the paper, we
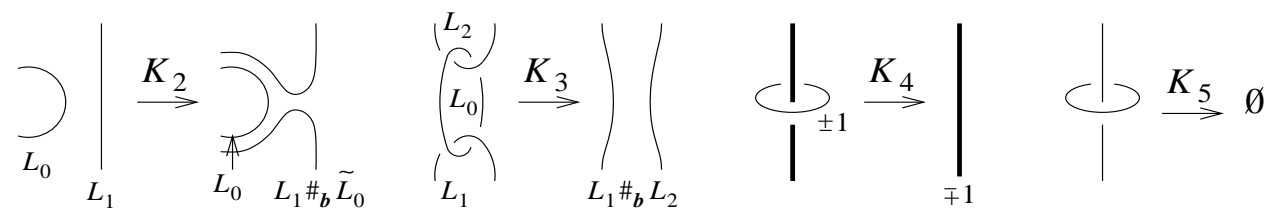

Figure 2: Kirby moves

think about a surgery presentation of $M$ and use thick solid lines to depict an arbitrary union of surgery components comprising $M$ (possibly together with some edges of embedded graphs). Also, we use a usual convention that all depicted links, or graphs, coincide outside a ball shown in the picture. In all figures the framings are assumed to be orthogonal to the plane of the picture, unless explicitly indicated otherwise. Some examples of the moves $K_{2}, K_{4}$ and $K_{5}$ are given in Figure 3. By a repeated application of $K_{3}$ we obtain the
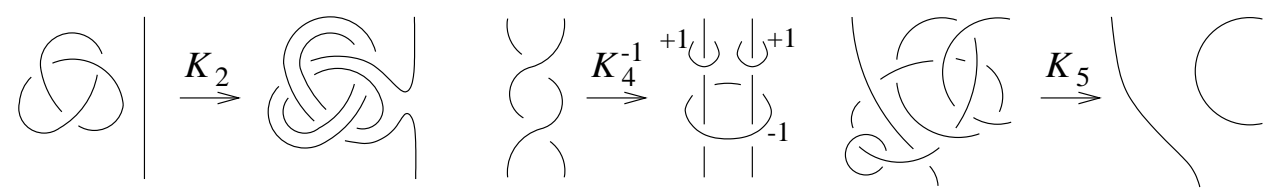

Figure 3: Examples of Kirby moves

following relation (which explains the term Borromean surgery, used in [10] for $\mathrm{Y}$-surgery in a manifold $M$, and the term $\Delta$-move, used in [12]):

\section{Lemma 2.1}
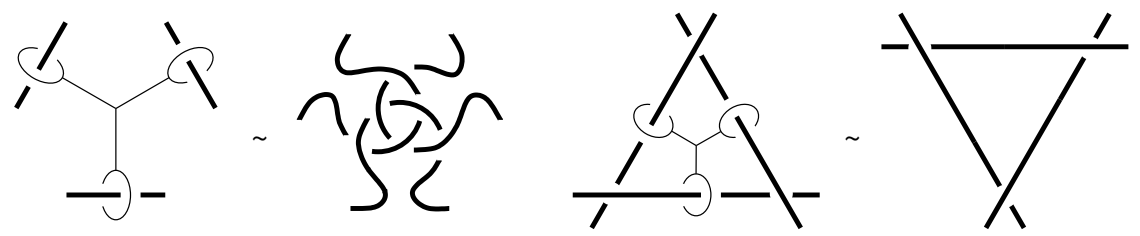

Geometry 85 Topology, Volume 5 (2001) 


\subsection{Clovers}

Let $G$ be a framed trivalent graph, smoothly embedded into a manifold $M$. We will call $G$ a clover, if:

- Each point of $G$ has a neighborhood, diffeomorphic to a neighborhood of some point of a standard Y-graph (including the framing), by an orientation-preserving diffeomorphism.

- Different edges meeting in a vertex have different tangent lines.

If both endpoints of an edge coincide, the two corresponding tangent lines may coincide. In this case the edge is called a leaf, and the incident vertex is called an external vertex. A vertex not incident to a leaf is internal. We call an edge internal, if both its endpoints are internal vertices. A degree of $G$ is the number of internal vertices. For example, a Y-graph is a degree 1 clover. It has one internal vertex, three external vertices and three leaves, see Figure 1a.

Clovers of degree 0 play an important role in an analogous theory of finite type invariants of links. However, we will exclude them from the theory of finite type invariants of 3-manifolds. Therefore all throughout the paper we will always assume that each connected component of a clover is of degree at least one.

In figures we will always assume that the framing of $G$ is orthogonal to the plane of the picture.

\section{$2.3 \quad$ Surgery on clovers}

Let $G$ be a clover in $M$. We construct a framed link $L(G)$ in a small neighborhood of $G$. In Figure 4 we show the structure of $L(G)$ near an edge, a leaf, and an internal vertex of $G$. The framings of the fragments of $G$ and $L(G)$ appearing in Figure 4 are assumed to be orthogonal to the plane of the picture. The construction of $L(G)$ is illustrated in Figure 5. The surgery of $M$ along

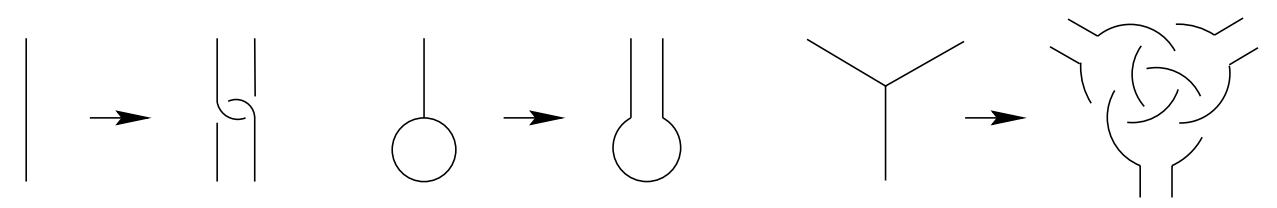

Figure 4: Construction of the surgery link from a clover

$L(G)$ is called a surgery along $G$, and the manifold $M_{L(G)}$ is denoted by $M_{G}$.

Geometry $\&$ Topology, Volume 5 (2001) 


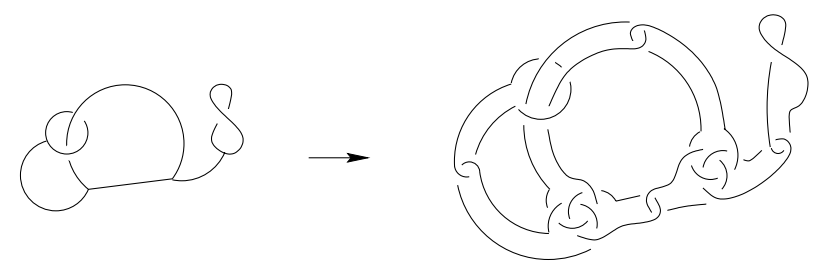

Figure 5: A clover and the corresponding link

We call two clovers $G$ and $G^{\prime}$ in $M$ surgery equivalent, and denote $G \sim G^{\prime}$, if $M_{G}$ is diffeomorphic to $M_{G^{\prime}}$.

Denote by $G_{1}, \ldots, G_{n}$ the connected components of $G$. For an $n$-tuple $\sigma=$ $\left\{\sigma_{1}, \ldots, \sigma_{n}\right\} \in\{0,1\}^{n}$ we put

$$
[M, G]=\sum_{\sigma}(-1)^{|\sigma|} M_{G(\sigma)},
$$

where $G(\sigma)=\bigcup_{i: \sigma_{i}=1} G_{i}$ and $|\sigma|=\sum_{i} \sigma_{i}$. If the degree of each $G_{i}$ is 1 , we recover the definition of $[M, G]$ for $\mathrm{Y}$-links.

Suppose that a leaf or a cycle of edges of $G$ in $M$ bounds an embedded surface $\Sigma$ in $M$. Then the intersection number of its push-off along the framing with $\Sigma$ does not depend on the choice of $\Sigma$ and (by a slight abuse of terminology) will be also called the framing of this leaf or cycle. A leaf is trivial, if it is 0 -framed and bounds a disc $D$ in $M$ whose interior does not intersect $G$.

Lemma 2.2 Let $G \subset M$ be a connected clover, which contains a trivial leaf $l$. Then surgery on $G$ preserves a neighborhood of $G \cup D$, where $D$ is the disc bounding $l$. In particular, $M_{G}=M$ and $[M, G]=0$ :

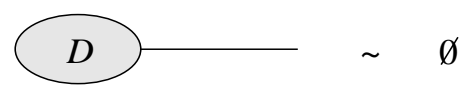

Proof Follows from the construction of $L(G)$ by an application of $K_{5}$.

An edge of a clover is a trivial loop, if both its endpoints coincide, it is 0-framed, and bounds a disc $D$ whose interior does not intersect $G$.

Lemma 2.3 Let $G \subset M$ be a connected clover, which contains a trivial looped edge $e$. Then surgery on $G$ preserves a neighborhood of $G \cup D$, where $D$ is the disc bounding $e$. In particular, $M_{G}=M$ and $[M, G]=0$. 
Proof Follows from the construction of $L(G)$ by an application of $K_{5}$ :

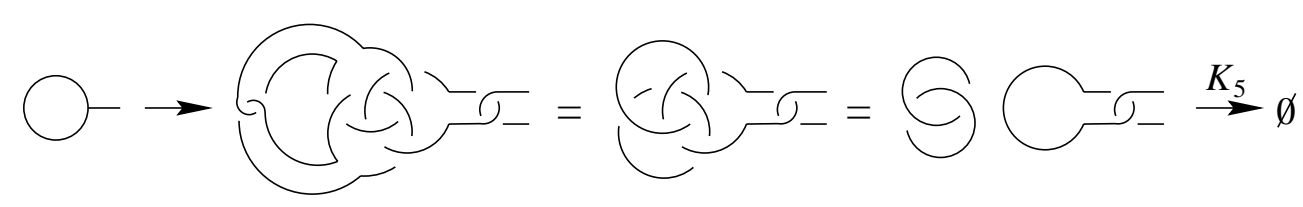

Theorem 2.4 Let $G$ be a clover in $M$, and let $G^{\prime}$ be obtained from $G$ by cutting an internal edge of $G$ and inserting there two small Hopf-linked leaves:

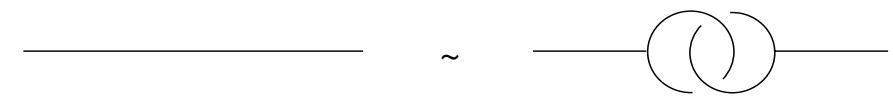

Then $G^{\prime} \sim G$ and $\left[M, G^{\prime}\right]= \pm[M, G]$, where the sign is negative if the edge is splitting $G$, and positive otherwise.

Proof The equality $G \sim G^{\prime}$ follows from the construction of $L(G)$ by an application of $K_{3}$. Topologically, this corresponds to cutting the corresponding solid handlebody introducing a pair of complimentary handles as shown below:

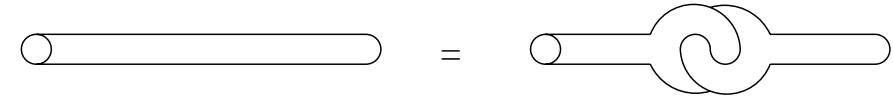

Let $G_{0}$ be the component of $G$ containing this edge, and $G_{1}, G_{2}$ be the components of $G^{\prime}$ replacing $G_{0}$. Note that each subclover of $G^{\prime}$ which contains exactly one of the components $G_{1}$ and $G_{2}$, has a trivial leaf. Thus by the definition of $[\cdot, \cdot]$ and Lemma $2.2\left[M, G^{\prime}\right]=[M, G]$ if $G^{\prime}$ has the same number of components as $G$, and $\left[M, G^{\prime}\right]=-[M, G]$ otherwise.

Corollary 2.5 Let $G$ be a degree $n$ clover. Then $[M, G] \in \mathcal{F}_{n}^{Y}(M)$.

\section{Topological calculus of clovers}

Below we describe some important moves $Y_{1}-Y_{4}$ on $\mathrm{Y}$-links, which are shown to preserve the surgery equivalence classes. One can apply these moves also to clovers, in view of Theorem 2.4.

Moves $Y_{1}$ and $Y_{2}$ (similar to the Kirby moves $K_{1}, K_{2}$ ) are shown in Figure 6:

$Y_{1}$ : Add to $G$ a connected clover $G^{\prime}$, which has a 0 -framed leaf $l$ bounding an embedded disc whose interior does not intersect $G \cup G^{\prime}$. 

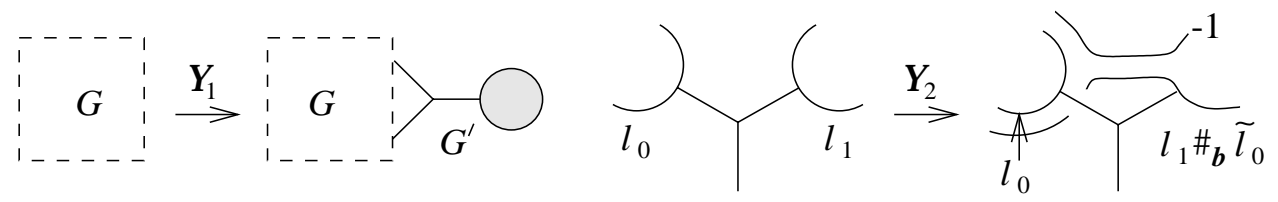

Figure 6: Blow-up and leaf slide

$Y_{2}$ : Add a leaf $l_{0}$ to another leaf $l_{1}$ of the same Y-graph along a band $b$ by replacing $l_{1}$ with $l_{1} \#_{b} \widetilde{l_{0}}$, where $\widetilde{l_{0}}$ is a push-off of $l_{0}$ along the framing; then change the framing by -1 .

Let $l$ be a leaf of a Y-graph $G_{0}$ and $e$ be the adjacent edge. Let $K$ be a knot. The move $Y_{3}$ is sliding an edge $e$ along $K$, see Figure 7:

$Y_{3}$ : Denote by $G_{1} \mathrm{Y}$-graph obtained from $G_{0}$ by adding $K$ to $e$ along a band $b$. Construct a $Y$-graph $G_{2}$ as shown in Figure 7: one of its leaves is a push-off of $K$ (and the adjacent edge goes along $b$ ), another is a push-off of $l$, and the third leaf is a 0 -framed unknot linked once with $l$. Replace $G_{0}$ by $G_{1} \cup G_{2}$.
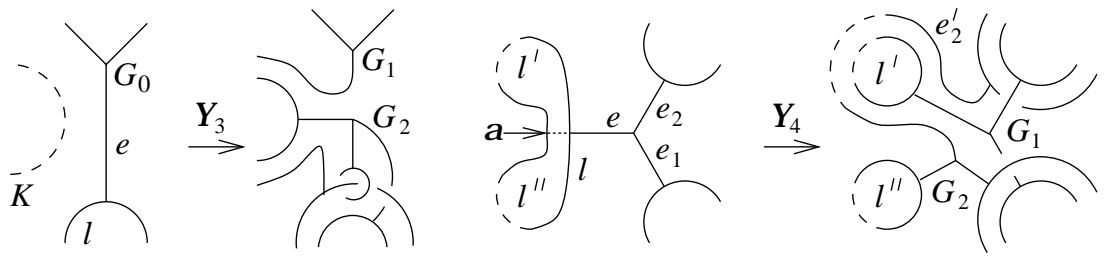

Figure 7: Edge slide and leaf-cutting

Let $l$ be a leaf of a Y-graph $G_{0}, e, e_{1}$ and $e_{2}$ be its edges, with $e$ adjacent to $l$. An arc $a$, starting at $e \cap l$ and ending on $l$, cuts $l$ into two parts $l^{\prime}$ and $l^{\prime \prime}$. The move $Y_{4}$ is cutting the leaf $l$ along $a$, see Figure 7 :

$Y_{4}$ : Let $G_{1}$ be an Y-graph obtained from $G_{0}$ by replacing the leaf $l$ by $l^{\prime} \cup a$. Let $G_{2}$ be obtained from $G_{0}$ by replacing the leaf $l$ by $l^{\prime \prime} \cup a$, adding $l^{\prime}$ to $e_{2}$ along the band $e$, and taking a push-off copy $e_{2}^{\prime}$, as shown in Figure 7. Replace $G_{0}$ by $G_{1} \cup G_{2}$.

A convenient way to draw the moves $Y_{1}-Y_{4}$ is by depicting them in a standard handlebody, which then can be embedded into a manifold in an arbitrary way. Below is such an interpretation of the moves $Y_{3}, Y_{4}$ as moves in a standard handlebody of genus 4: 

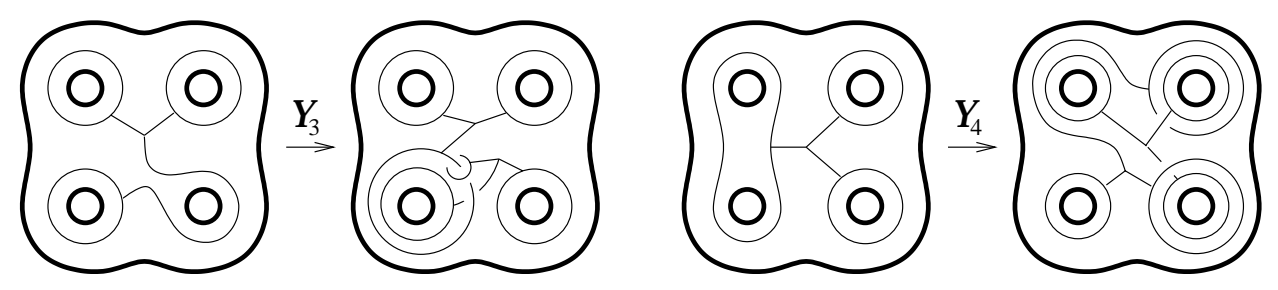

Figure 8: $Y_{3}$ and $Y_{4}$ as moves in a handlebody

For example, when the handlebody is embedded so that one of the handles links another handle as in Figure 9a, the corresponding move $Y_{4}$ is shown in Figure $9 \mathrm{~b}$.
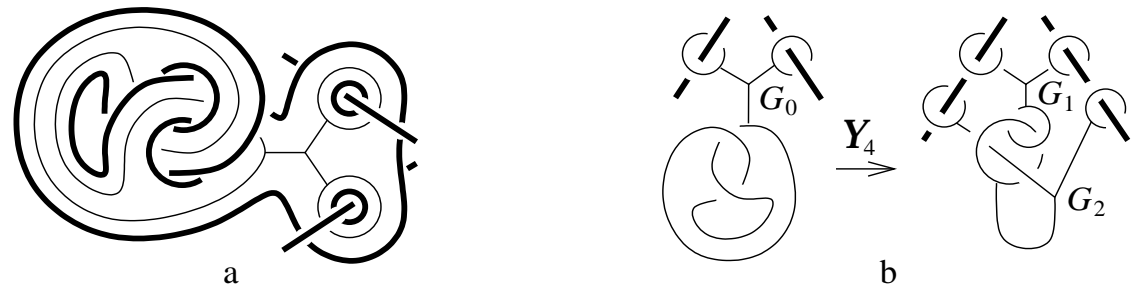

Figure 9: An embedding and the induced $Y_{4}$ move

Theorem 3.1 The moves $Y_{1}-Y_{4}$ preserve the classes of surgery equivalence of $Y$-links in a manifold $M$.

Proof It suffices to prove the surgery equivalence of $\mathrm{Y}$-links obtained by the moves $Y_{1}-Y_{4}$ in the standard handlebody. Instead of drawing the handlebodies we will draw thick lines passing through the handles (encoding a set of surgery and clover components), similarly to Lemma 2.1.

By Lemma 2.2, $Y_{1}$ is surgery equivalence. To verify $Y_{2}$, depict the Borromean linking with one component passing on the boundary of an embedded surface with two handles, and then twist one of the handles along the other:

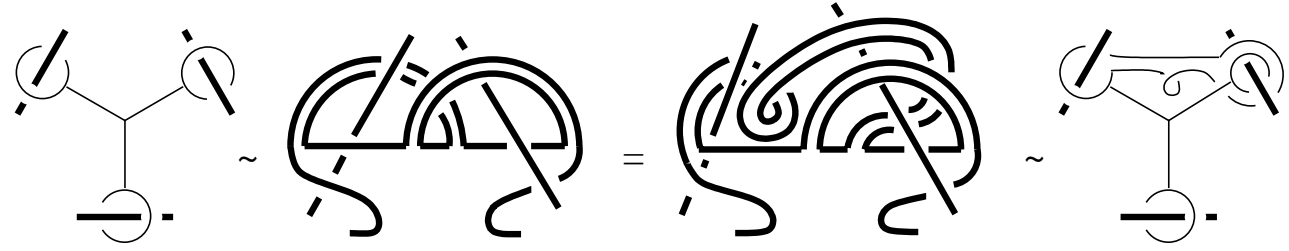

Geometry \& Topology, Volume 5 (2001) 
To verify $Y_{3}$, use an isotopy and Lemma 2.1 :

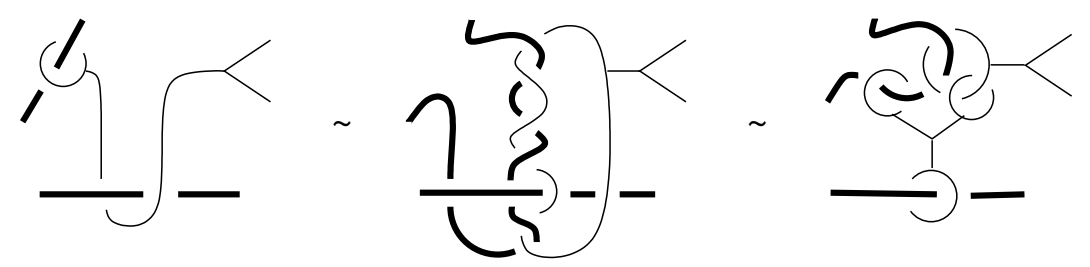

The verification of $Y_{4}$ is similar:

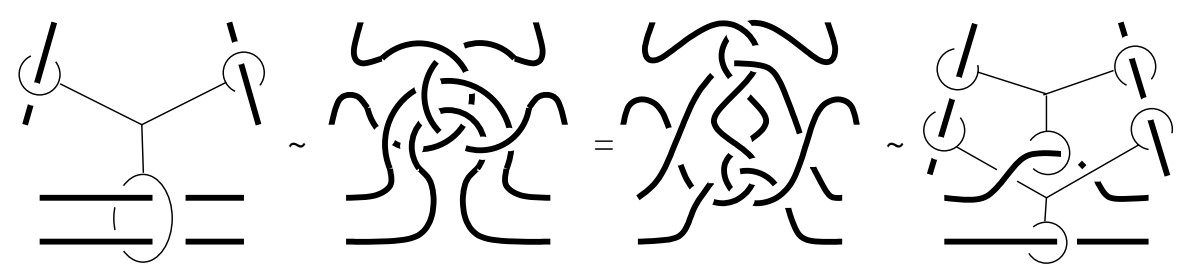

The following theorem was announced in [5] in case of a Y-graph $G$.

Theorem 3.2 For any clover $G$ in a manifold $M$, there exists a clover $G^{-1}$ in a neighborhood of $G$, such that the result of surgery on both clovers is the original manifold: $M_{G \cup G^{-1}}=M$. The construction of $G^{-1}$ for a $Y$-graph $G$ is shown in Figure 10a. Another presentation of $G^{-1}$ by a 2-component $Y$-link $G_{1} \cup G_{2}$ is shown in Figure 10b.

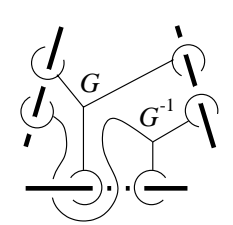

a

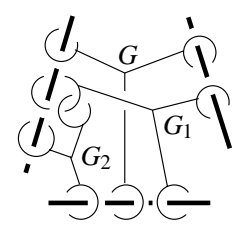

b

Figure 10: Two presentations of the inverse of a Y-graph

Proof Let us first verify the statement for a $\mathrm{Y}$-graph. By an isotopy and a subsequent application of $Y_{4}$ and $Y_{1}$, we get
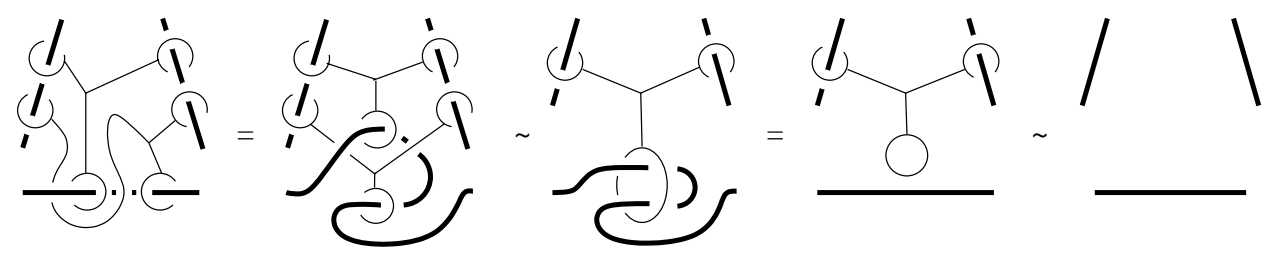

Geometry \& $\mathcal{T}$ Topology, Volume 5 (2001) 
The general case now follows by Theorem 2.4. Finally, $G^{-1} \sim G_{1} \cup G_{2}$ by $Y_{3}$.

\section{The structure of the graded spaces $\mathcal{G}_{n}$}

This section is devoted to the study of the graded spaces

$$
\mathcal{G}_{n}(M)=\mathcal{F}_{n}^{Y}(M) / \mathcal{F}_{n+1}^{Y}(M) .
$$

Denote $[M, G] \underset{n}{=}\left[M, G^{\prime}\right]$ iff $[M, G]-\left[M, G^{\prime}\right] \in \mathcal{F}_{n+1}^{Y}(M)$.

\subsection{Graded versions of $Y_{2}-Y_{4}$}

Using 2.4 and Lemma 2.2, we obtain the following immediate corollaries of Theorem 3.1.

Corollary 4.1 Let $G$ be a clover of degree $n$ in a manifold $M$, and let $G^{\prime}$ be obtained from $G$ by sliding one of its leaves along an adjacent leaf by $Y_{2}$. Then $\left[M, G^{\prime}\right]=[M, G]$, and hence $\left[M, G^{\prime}\right] \underset{n}{=}[M, G]$.

Let $G_{0}$ be a Y-graph in $M$, and let $G_{1} \cup G_{2}$ be obtained from $G_{0}$ by an application of $Y_{3}$, see Figure 7 . Note that $M_{G_{2}}=M$ by $Y_{1}$, thus

$$
\left[M, G_{0}\right]-\left[M, G_{1}\right]=M_{G_{1} \cup G_{2}}-M_{G_{1}}=\left[M, G_{1} \cup G_{2}\right] \in \mathcal{F}_{2}^{Y}(M) .
$$

Hence, using Theorem 2.4 we obtain:

Corollary 4.2 Let $G$ be a clover of degree $n$ in a manifold $M$, and let $K \subset M$ be a knot. Let $G^{\prime}$ be obtained from $G$ by sliding an edge of $G$ along $K$. Then $\left[M, G^{\prime}\right] \underset{n}{=}[M, G]$.

In Lemma 4.10 we will strengthen this result by computing the difference $\left[M, G^{\prime}\right]-[M, G]$ in $\mathcal{G}_{n+1}(M)$.

Let $G_{0}$ be a Y-graph in $M$, and let $G_{1} \cup G_{2}$ be obtained from $G_{0}$ by an application of $Y_{4}$, see Figure 7 . By the previous Lemma, the value of $\left[M, G_{2}\right]$ in $\mathcal{G}_{1}(M)$ does not change when we slide its edge $e_{2}^{\prime}$ along a knot. Thus we can pull $e_{2}^{\prime}$ off $l^{\prime}$, making it back into $e_{2}$. Hence, using again Theorem 2.4 we obtain: 
Corollary 4.3 Let $G$ be a clover of degree $n$ in a manifold $M$ and $l$ be a leaf of $G$. An arc a starting in the external vertex incident to $l$ and ending in other point of $l$, splits $l$ into two arcs $l^{\prime}$ and $l^{\prime \prime}$. Denote by $G^{\prime}$ and $G^{\prime \prime}$ the clovers obtained from $G$ by replacing the leaf $l$ with $l^{\prime} \cup a$ and $l^{\prime \prime} \cup a$ respectively, see Figure 11. Then $[M, G] \underset{n}{=}\left[M, G^{\prime}\right]+\left[M, G^{\prime \prime}\right]$.
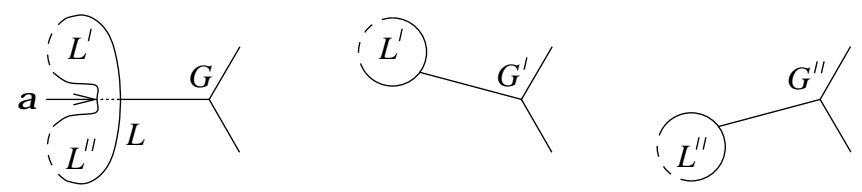

Figure 11: Splitting a leaf

\subsection{The dependence on framings}

Theorem 3.2 allows us to deduce the dependence of $[M, G]$ on the framings of edges.

Lemma 4.4 Let $G$ be a clover of degree $n$ in a manifold $M$. Let $G^{\prime}$ be obtained from $G$ by twisting the framing of an edge by a half twist. Then $\left[M, G^{\prime}\right] \underset{n}{=}-[M, G]$.

Proof Let $G$ and $G_{1} \cup G_{2} \sim G^{-1}$ be the Y-graphs depicted in Figure 10b. Note that $G_{1}$ looks exactly like $G$, except for the way its lower leaf links the thick line. Turning this leaf to the same position changes the framing of the adjacent edge by a half twist:

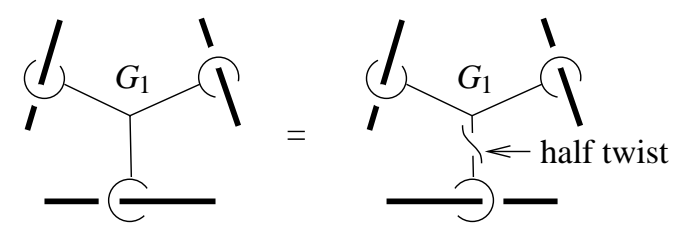

By Theorem 3.2, $M_{G \cup G_{1} \cup G_{2}}=M$; also, $M_{G \cup G_{2}}=M_{G}$ and $M_{G_{2}}=M$ by Lemma 2.2. Thus

$$
\begin{aligned}
{[M, G]+\left[M, G_{1}\right] } & =2 M-M_{G}-M_{G_{1}} \\
& =\left[M, G \cup G_{1}\right]+\left[M, G_{1} \cup G_{2}\right]-\left[M, G \cup G_{1} \cup G_{2}\right]=0
\end{aligned}
$$

and the lemma follows. 
Alternatively, one can show that $[M, G]+\left[M, G^{-1}\right]=0$ for a Y-graph $G^{-1}$ depicted in Figure 10a and pull its edge off the lower thick line by 4.2 to obtain once again $G_{1}$.

Corollary 4.5 Let $G$ be a clover of degree $n$ in $M$. Adding a kink, ie, a full twist to the framing of an edge preserves an $n$-equivalence class of $[M, G]$.

Now, note that the following two $\mathrm{Y}$-graphs are isotopic:

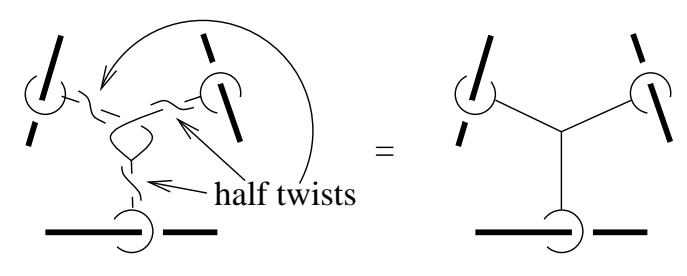

Thus, applying Lemma 4.4 and Theorem 2.4, we obtain:

Corollary 4.6 Let $G$ and $G^{\prime}$ be clovers of degree $n$ in a manifold $M$, which coincide everywhere except for a fragment shown in Figure 12. Then $\left[M, G^{\prime}\right] \underset{n}{=}$ $-[M, G]$.
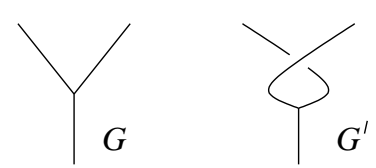

Figure 12: The AS relation

\subsection{Simplifying the leaves}

Denote $\mathcal{G}_{n}\left(S^{3}\right)$ by $\mathcal{G}_{n}$. We want to show that the space $\mathcal{G}_{n} \otimes \mathbb{Z}[1 / 2]$ is generated by clovers with only internal vertices, ie, without leaves.

A leaf of a clover $G$ is special, if it is \pm 1 -framed and bounds an embedded disc, whose interior does not intersect $G$. A leaf $l$ of a clover $G$ is simple, if it either bounds an embedded $i$-framed disc $D$ whose interior intersects $G$ in at most one point, or is special.

For $M=S^{3}$, the graded space $\mathcal{G}_{n}$ is generated by clovers of degree $n$ all leaves of which are simple. Indeed, suppose we are given an arbitrary clover of degree 
$n$ in $S^{3}$. We may split each of its non-trivial leaves into small pieces and apply Corollary 4.3 to present $G$ in $\mathcal{G}_{n}$ as a linear combination of clovers with simple leaves.

Suppose that all leaves of clover $G$ in a manifold $M$ are simple. If two simple leaves are linked with each other (see Figure 13a), we replace them by a new edge by Theorem 2.4. Suppose that at least one simple leaf $l$ still remains after this procedure. We will show below that in this case $[M, G] \in \mathcal{F}_{n+1}^{Y}(M) \otimes \mathbb{Z}[1 / 2]$.

We should consider three cases, see Figure 13b-d. Firstly, it may be that $l$ is trivial. Secondly, it may be that $l$ is 0 -framed, and the disc $D$ intersects an edge of $G$. Thirdly, it may be that $l$ is special. In the first case, $[M, G]=0$ by
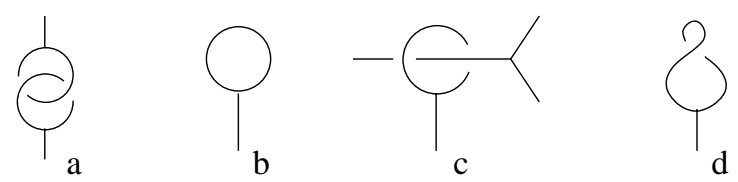

Figure 13: Four types of simple leaves

Lemma 2.2. The second case can be reduced to the first case after unlinking the edge from the corresponding leaf by Corollary 4.2 (sliding the edge along a small unknot linked once with the leaf). Thus we readily obtain:

Lemma 4.7 Let $G$ be a clover of degree $n$ in a manifold $M$. Suppose that $G$ contains a 0 -framed leaf bounding an embedded disc, whose interior intersects $G$ in exactly one point, belonging to an edge. Then $[M, G] \underset{n}{=} 0$.

In the third case we encounter 2 -torsion.

Lemma 4.8 Let $G$ be a clover of degree $n$ in a manifold $M$, which contains a special leaf. Then $2[M, G] \underset{n}{=} 0$.

Proof Rotating the special leaf we can change the framing of the adjacent edge while preserving the isotopy class of $G$. Thus by Lemma $4.4[M, G] \underset{n}{=}-[M, G]$ and the lemma follows.

Over the integers, we have the following inclusion:

Lemma 4.9 Let $G$ and $l$ be as in Lemma 4.8. Suppose that the connected component of $G$ which contains $l$ is of degree at least two. Then $[M, G] \underset{n}{=} 0$. 
Proof Cutting the neighboring internal edge we obtain two new leaves $l_{1}$ and $l_{2}$. Without a loss of generality suppose that the framing of $l$ is +1 . Then slide $l$ along $l_{1}$ by $Y_{2}$ as shown below:

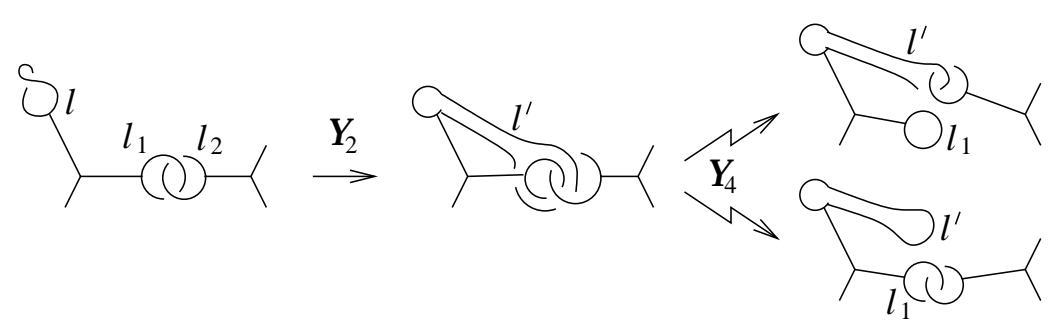

Notice that $Y_{2}$ changes the framing of a leaf by -1 , so the new leaf $l^{\prime}$ is 0 framed. Splitting $l_{2}$ as shown above by Corollary 4.3, we obtain two clovers each of which contains a trivial leaf (either $l^{\prime}$ or $l_{1}$ ), and so can be removed by $Y_{1}$.

\subsection{The IHX relation}

Let $G=\Gamma \cup G_{0}$ be an $n$-component Y-link which contains a Y-graph $G_{0}$, and let $K$ be a knot in $M$. Choose a band connecting an edge of $G_{0}$ to $K$. Sliding this edge of $G_{0}$ along $K$ by $Y_{3}$, we obtain a $\mathrm{Y}$-graph $G_{1}$, as shown in Figure 7. Denote $G^{\prime}=\Gamma \cup G_{1}$. By Corollary 4.2, $[M, G]-\left[M, G^{\prime}\right] \in \mathcal{F}_{n+1}^{Y}(M)$. There is a simple expression for this difference modulo $\mathcal{F}_{n+2}^{Y}(M)$. It is easier to visualize the picture in a neighborhood $N$ of $G_{0} \cup b \cup K$, which is a genus 4 handlebody embedded into $M$.

Lemma 4.10 Let $G_{0}, G_{1}$ and $G_{H}$ be the clovers of Figure 14 in a handlebody $V$ embedded into $M$. Let $\Gamma$ be a degree $n-1$ clover in the complement of $N$. Put $G=\Gamma \cup G_{0}, G^{\prime}=\Gamma \cup G_{1}$ and $G^{\prime \prime}=\Gamma \cup G_{H}$. Then $[M, G]-\left[M, G^{\prime}\right] \underset{n+1}{=}$ $\left[M, G^{\prime \prime}\right]$.
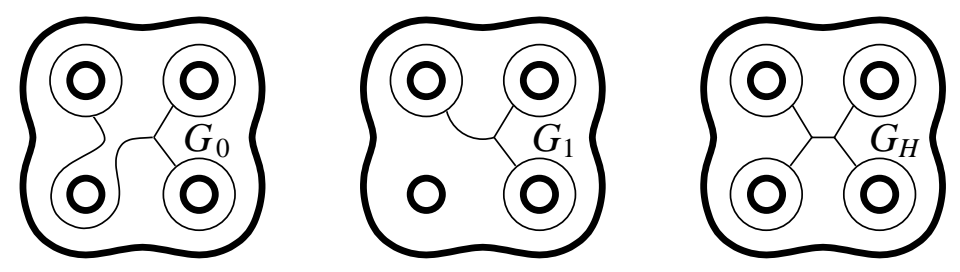

Figure 14: Sliding an edge and computing the difference 
Here is another graphical expression for the above graphs:
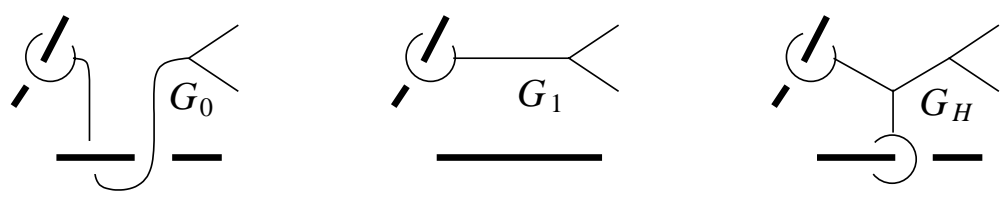

Proof We use $Y_{3}$ to pass from $G_{0}$ to $G_{1}$ and $G_{2}$ with $G_{0} \sim G_{1} \cup G_{2}$ as in the proof of Theorem 3.1. Then we split the leaf $l$ of $G_{1}$ by Corollary 4.3 introducing new Y-graphs $G_{1}^{\prime}$ and $G_{1}^{\prime \prime}$ with $\left[M, \Gamma \cup G_{1} \cup G_{2}\right] \underset{n+1}{=}\left[M, \Gamma \cup G_{1}^{\prime} \cup\right.$ $\left.G_{2}\right]+\left[M, \Gamma \cup G_{1}^{\prime \prime} \cup G_{2}\right]:$

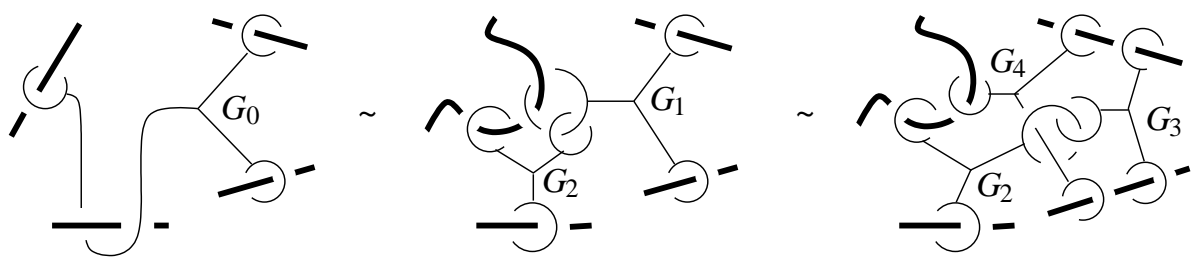

But $\left[M, \Gamma \cup G_{1}^{\prime \prime} \cup G_{2}\right]=-\left[M, G^{\prime \prime}\right]$ by Theorem 2.4, and $\left[M, \Gamma \cup G_{1}^{\prime} \cup G_{2}\right]=0$ by Lemma 2.2. Hence $\left[M, \Gamma \cup G_{1} \cup G_{2}\right] \underset{n+1}{=}-\left[M, G^{\prime \prime}\right]$. On the other hand,

$$
\left[M, \Gamma \cup G_{1} \cup G_{2}\right]=\sum_{\Gamma^{\prime} \subset \Gamma}(-1)^{\left|\Gamma^{\prime}\right|}\left(-M_{\Gamma^{\prime} \cup G_{1}}+M_{\Gamma^{\prime} \cup G_{1} \cup G_{2}}\right)=\left[M, G^{\prime}\right]-[M, G]
$$

The comparison of two above expressions for $\left[M, \Gamma \cup G_{1} \cup G_{2}\right]$ proves the theorem.

Theorem 4.11 Let $G_{I}, G_{H}$ and $G_{X}$ be clovers of degree $n$ in a manifold $M$, which coincide everywhere except for a fragment shown in Figure 15. Then $\left[M, G_{I}\right]+\left[M, G_{X}\right]-\left[M, G_{H}\right] \underset{n}{=} 0$.
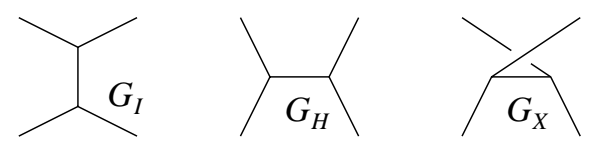

Figure 15: The IHX relation

Proof It suffices to prove the statement for $n=2$; the general case then follows by Theorem 2.4 and Lemma 2.2. Consider a standard Y-graph $G$ in a handlebody $N$ of genus 3 and attach to $N$ an additional handle $h$. We are to slide all three edges of $Y$ along $h$ in a genus 4 handlebody $N \cup h$ as indicated 

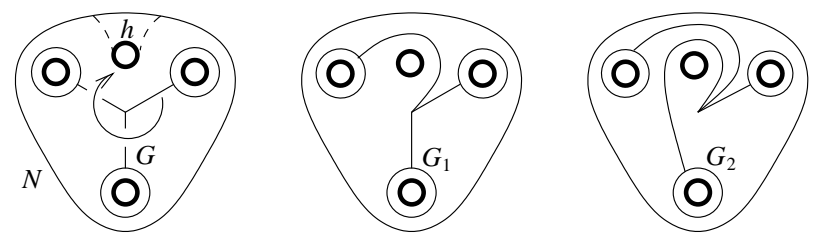

Figure 16: Sliding the edges along a handle

in Figure 16. Each time we will use Lemma 4.10 to compute the corresponding change of $[M, G]$. Sliding the first edge of $G$, we obtain a new Y-graph $G_{1}$ with $\left[M, G_{1}\right]-[M, G]=\frac{\overline{2}}{2}\left[M, G_{I}\right]$. Sliding the next edge of $G$ (or rather of $G_{1}$ ), we obtain a new Y-graph $G_{2}$ with $\left[M, G_{2}\right]-\left[M, G_{1}\right]=\left[M, G_{X}^{\prime}\right]$. After sliding the third edge we return back to $G$ and get $[M, G]-\left[M, G_{2}\right] \underset{2}{\overline{2}}\left[M, G_{H}^{\prime}\right]$. Here a degree 2 clovers $G_{I}, G_{X}^{\prime}$, and $G_{H}^{\prime}$ are shown in Figure 17. Summing up these

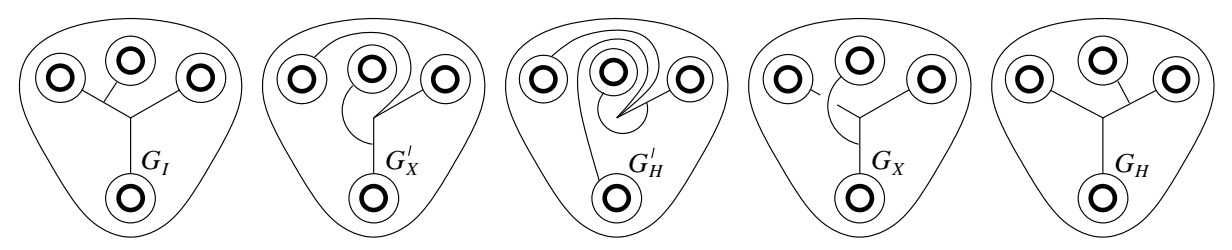

Figure 17: Computing the difference

three equalities we get

$$
\left[M, G_{I}\right]+\left[M, G_{X}^{\prime}\right]+\left[M, G_{H}^{\prime}\right]=\frac{\overline{2}}{2} 0
$$

But $G_{X}^{\prime}$ differs from $G_{X}$ only by an edge slide, and $G_{H}^{\prime}$ differs from $G_{H}$ by an edge slide and a cyclic ordering of edges in a vertex, see Figure 17. Hence $\left[M, G_{X}^{\prime}\right]=\left[\overline{\overline{2}}\left[M, G_{X}\right]\right.$ by Corollary 4.2 , and $\left[M, G_{H}^{\prime}\right] \overline{\overline{2}}-\left[M, G_{H}\right]$ by Corollaries 4.2 and 4.6. A substitution of two these expressions into (1) proves the theorem.

Remark 4.12 There is a topological version of the IHX relation, which may be deduced similarly using the $Y_{3}$ and $Y_{4}$ moves to slide each edge of a Ygraph through a handle in a handlebody of genus 4 . We leave the details to an interested reader. 


\subsection{Trivalent graphs and $\mathcal{G}_{n}$}

Consider an abelian group $\widetilde{\mathcal{A}}_{k}$ freely generated by abstract (not necessarily connected) trivalent graphs with $2 k$ vertices and without looped edges, equipped with a cyclic ordering of the incident edges in each vertex. Denote by $\mathcal{A}_{k}$ the quotient of $\widetilde{\mathcal{A}}_{k}$ by the following AS and IHX relations:

AS: Let $G^{\prime}$ be obtained from $G$ by reversing the cyclic ordering of edges in some vertex, see Figure 12. Then $G^{\prime}=-G$.

IHX: Let $G_{I}, G_{H}$ and $G_{X}$ coincide everywhere except for a fragment shown in Figure 15. Then $G_{I}=G_{H}-G_{X}$.

Denote by $Y$ a $\mathrm{Y}$-graph in $M$ with three special leaves. For each graph $G \in \widetilde{\mathcal{A}}_{k}$ pick an arbitrary embedding of $G$ into $M$. Equip it with a framing so that the framing along each cycle of edges is integer, and take its disjoint union with $m$ copies of $Y$. The resulting framed graph in $M$ may be considered as a clover of degree $n=2 k+\underset{\widetilde{\mathcal{A}}}{\sim}$. Denote it by $\phi(G)$. Put $\widetilde{\psi}_{n}(G)=[M, \phi(G)]$ and extend $\widetilde{\psi}_{n}$ to $\widetilde{\psi}_{n}: \oplus_{2 k \leq n} \widetilde{\mathcal{A}}_{k} \rightarrow \mathcal{F}$ by linearity. Note that $\widetilde{\psi}_{n}(\widetilde{\mathcal{A}}) \subset \mathcal{F}_{n}^{Y}$ by Corollary 2.5 .

Theorem 4.13 The map $\widetilde{\psi}_{n}: \oplus_{2 k \leq n} \widetilde{\mathcal{A}}_{k} \rightarrow \mathcal{F}_{n}^{Y}$ induces a quotient map $\psi_{n}: \oplus_{2 k \leq n} \mathcal{A}_{k} \rightarrow \mathcal{G}_{n}$, which is surjective and does not depend on the choice of $\phi$. The image of $\oplus \mathcal{A}_{2 k<n}$ is a 2 -torsion.

Proof The map $\widetilde{\psi}_{n}$ factors through AS and IHX relations by Corollary 4.6 and Theorem 4.11. The independence on the choice of $\phi(G)$ follows from Corollary 4.2 and Lemma 4.4. The surjectivity follows from the results of Section 4.3 and Lemma 2.3. The torsion result follows from Lemma 4.8.

Note that $\oplus_{2 k \leq 2 n-1} \mathcal{A}_{k}=\oplus_{2 k<2 n-1} \mathcal{A}_{k}$, hence $\mathcal{G}_{2 n-1}$ consists of a 2 -torsion and we obtain:

Corollary 4.14 For any $n \in \mathbb{N}, \mathcal{F}_{2 n-1}^{Y} \otimes \mathbb{Z}[1 / 2]=\mathcal{F}_{2 n}^{Y} \otimes \mathbb{Z}[1 / 2]$.

\section{The equivalence of the $\mathcal{F}^{b l}, \mathcal{F}^{a s}$ and $\mathcal{F}^{Y}$ filtrations}

\subsection{Equivalence of $\mathcal{F}^{b l}$ and $\mathcal{F}^{Y}$}

We can present surgery on an arbitrary blink of genus $g$ as surgery on $g$ blinks of genus one, slicing the surface into pieces of genus one as shown below (see 
also [3]):

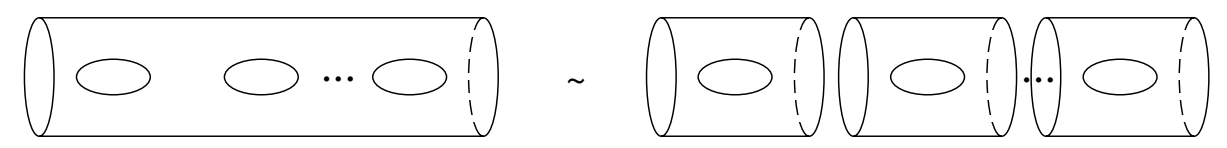

Also, as noticed already in [10], surgery on a blink of genus one can be presented as $\mathrm{Y}$-surgery (and vice versa). Indeed, depicting the Borromean linking with one component passing on the boundary of an embedded surface with two bands, and then using $K_{3}^{-1}$, we obtain:

\section{Lemma 5.1}

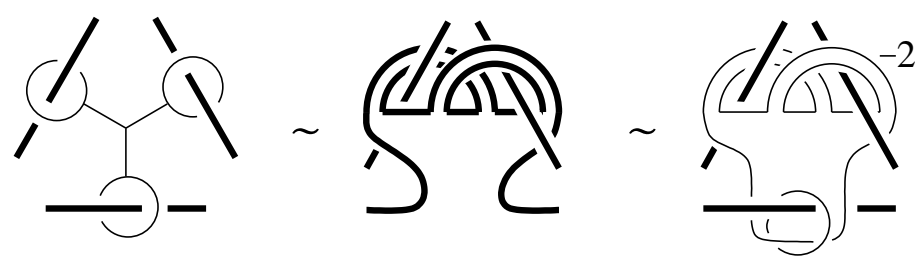

Therefore the following theorem holds:

Theorem 5.2 For each integer $n$ we have $\mathcal{F}_{n}^{b l}(M)=\mathcal{F}_{n}^{Y}(M)$.

\subsection{Comparison of $\mathcal{F}^{a s}$ and $\mathcal{F}^{Y}$ : plan of the proof}

The rest of the section is arranged in the following way.

We will first present any $\mathrm{Y}$-link of degree $d$ by a trivial $d$-component $\mathrm{Y}$-link in $S^{3}$, together with a trivial unimodular link $O$ which links $T$ in a special way. We shall then prove an inclusion $\mathcal{F}_{2 n}^{Y} \subset \mathcal{F}_{3 n}^{a s}$ by an easy counting argument.

The opposite inclusion is similar in spirit. Now we present any $n$-component algebraically split link by a trivial link $O$, together with a trivial $\mathrm{Y}$-link $T$ linking $O$ in a special way. We then consider an appropriately modified version of some results of Section 4. Building on these results, we prove the inclusion $\mathcal{F}_{3 n}^{a s} \subset \mathcal{F}_{2 n}^{Y} \otimes \mathbb{Z}[1 / 2]$ using a similar counting argument.

\subsection{Undoing $\mathrm{Y}$-graphs}

A link $O$ in $M \backslash G$ laces a clover $G$, if $O$ is trivial unimodular, and each of the (pairwise disjoint) discs bounding its components intersects $G$ in at most two points, which belong to the leaves of $G$. A Y-link in $M$ is trivial, if it consists of $\mathrm{Y}$-graphs standardly embedded in $n$ disjoint balls. 
Lemma 5.3 Let $T$ be a trivial $n$-component $Y$-link in $S^{3}$. For any $n-$ component $Y$-link $G$ in $S^{3}$, there exists a link $O$ in $S^{3}$ which laces $T$, such that $\left[S^{3}, G\right]=\left[S_{O}^{3}, T\right]$.

Proof Any Y-link in $S^{3}$, in particular $G$, can be made into a trivial Y-link by framing twists and crossing changes. Moreover, it suffices to use crossing changes which involve only the leaves of $\mathrm{Y}$-graphs. Indeed, instead of a crossing change which involves an edge of a $\mathrm{Y}$-graph, one can do two subsequent crossing changes with the neighboring leaf of this graph (by sliding first the other branch towards the leaf). Using $K_{4}$ we can realize each of these framing and crossing changes by surgery on a trivial unimodular surgery link, as illustrated in Figure 3. The resulting collection of these surgery components comprises $O$.

For a Y-link $G$ in a manifold $M$ and a link $L \subset M \backslash G$ denote by $[M, G, L]$ the double alternating sum:

$$
[M, L, G]=[[M, L], G]=[[M, G], L]=\sum_{G^{\prime} \subset G} \sum_{L^{\prime} \subset L}(-1)^{\left|G^{\prime}\right|+\left|L^{\prime}\right|} M_{G^{\prime} \cup L^{\prime}}
$$

Corollary 5.4 The space $\mathcal{F}_{n}^{Y}$ is generated by all $\left[S^{3}, O, T\right]$, where $T$ is a trivial $Y$-link in $S^{3}$ of degree at least $n$, and the link $O$ laces $T$.

Proof By Lemma 5.3, $\mathcal{F}_{n}^{Y}$ is generated by all $\left[S_{O}^{3}, T\right]$, with $O$ and $T$ as above. It remains to notice that $S_{O}^{3}=\sum_{O^{\prime} \subset O}(-1)^{\left|O^{\prime}\right|}\left[S^{3}, O^{\prime}\right]$ and that any sublink $O^{\prime}$ of $O$ also laces $T$.

Theorem 5.5 For each integer $n$ we have $\mathcal{F}_{2 n}^{Y} \subset \mathcal{F}_{3 n}^{a s}$.

Proof Let $T$ be a trivial $\mathrm{Y}$-link in $S^{3}$ of degree at least $2 n$ and $O$ be an arbitrary link lacing $T$. In view of Corollary 5.4, it suffices to prove that $\left[S^{3}, O, T\right]$ belongs to $\mathcal{F}_{3 n}^{a s}$. Suppose that some leaf of $T$ is not linked with $O$, ie, bounds a disc which does not intersect $O$; then $\left[S^{3}, O, T\right]=0 \in \mathcal{F}_{3 n}^{a s}$ by Lemma 2.2. Otherwise, all (ie, at least $6 n$ ) leaves of $T$ are linked with $O$. But each component of $O$ is linked with at most two leaves of $T$; hence the number of components of $O$ is at least $3 n$. Therefore $\left[S^{3}, O, T\right] \in \mathcal{F}_{3 n}^{a s}$. 


\subsection{Undoing an AS-link}

Let $L$ be a framed link in $S^{3}$. We call $L$ an $A S$-link, if it is algebraically split and unimodular. In Section 5.3 above we presented any Y-link by a trivial Y-link $T$, together with a trivial unimodular link $O$ lacing it. In this section we shall do the opposite: we present any AS-link by a trivial unimodular link $O$, together with a trivial $\mathrm{Y}$-link linking it in a special way, which we will also call "lacing".

A clover $G$ in $M \backslash L$ laces a link $L$, if each leaf of $G$ either is trivial and links $L$ once (ie, bounds a disc which intersects $L$ in one point), or is unlinked with $L$ (ie, bounds a surface which does not intersects $L) .^{2}$ A pair $(O, G)$ consisting of a trivial link $O$ in $M$ and a $\mathrm{Y}$-link $G$ lacing $O$ is called a lacing pair. A lacing pair $(O, G)$ is trivial, if $G$ is trivial. Surgery on a trivial lacing pair in $S^{3}$ was called a Borromean surgery in [10] and a $\Delta$-move in [12]. It was shown in $[10,12]$, that one can pass from a link $L$ in $S^{3}$ to any other link with the same linking matrix by surgery on a trivial $\mathrm{Y}$-link lacing $L$. Applying this result to AS-links, we deduce:

Lemma 5.6 Let $O$ be a trivial unimodular $n$-component link in a $S^{3}$. For any $A S$-link $L$ in $S^{3}$, there exists a trivial lacing pair $(O, G)$, such that $\left[S^{3}, L\right]=$ $\left[S_{T}^{3}, O\right]$.

Corollary 5.7 The space $\mathcal{F}_{n}^{a s}$ is generated by all $\left[S^{3}, O, T\right]$, where $O$ is a trivial unimodular link in $S^{3}$ with at least $n$ components and a trivial $Y$-link $G$ laces $O$.

Proof By Lemma 5.6, $\mathcal{F}_{n}^{\text {as }}$ is generated by all $\left[S_{T}^{3}, O\right]$, with $T$ and $O$ as above. It remains to notice that $S_{T}^{3}=\sum_{T^{\prime} \subset T}(-1)^{\left|T^{\prime}\right|}\left[S^{3}, T^{\prime}\right]$ and that any Y-sublink $T^{\prime}$ of $T$ also laces $O$.

In what follows, we will in fact need only a weaker version of Corollary 5.7, in which we omit the assumption of triviality of the lacing pair.

\subsection{The inclusion $\mathcal{F}_{3 n}^{a s} \subset \mathcal{F}_{2 n}^{Y} \otimes \mathbb{Z}[1 / 2]$}

We will need a modification of Corollary 4.2 and Lemma 4.4. Returning to their proofs, we notice that both statements can be stated for $\mathrm{Y}$-links lacing a fixed link in $M$ :

\footnotetext{
${ }^{2}$ In fact, we will not need this type of leaf, but prefer to formulate the definition in its full generality
} 
Corollary 5.8 Fix a link $L$ in a manifold $M$. Let $G$ be a $Y$-link of degree $d$ lacing $L$ and let $G^{\prime}$ be obtained from $G$ by sliding an edge of $G$ along a knot $K \subset M \backslash L \backslash G$. Suppose that for every $Y$-link $\Gamma$ of degree $(d+1)$ lacing $L$ one has $[M, L, \Gamma] \underset{k}{=} 0$. Then $\left[M, G^{\prime}\right] \underset{k}{=}[M, G]$.

Lemma 5.9 Fix a link $L$ in a manifold $M$. Let $G$ be a $Y$-link of degree $d$ lacing $L$ and let $G^{\prime}$ be obtained from $G$ by twisting the framing of some edge by a half twist. Suppose that for every $Y$-link $\Gamma$ of degree $(d+1)$ lacing $L$ one has $[M, L, \Gamma] \underset{k}{=} 0$. Then $\left[M, G^{\prime}\right] \underset{k}{=}-[M, G]$

Moreover, the following version of Lemma 4.8 holds:

Lemma 5.10 Let $(O, G)$ be a lacing pair in a manifold $M$ with $G$ of degree $d$. Suppose that some disc $D_{i}$ bounding a component of $O$ intersects $G$ in just one point, which belongs to a leaf of $G$. Suppose also that for any lacing pair $(O, \Gamma)$ with $\Gamma$ of degree greater than $d$ we have $[M, O, \Gamma] \underset{\bar{k}}{0} 0$. Then $2[M, G] \underset{k}{=} 0$.

Proof Proceeding similarly to the proof of Lemma 4.8, we rotate $O$ together with the trivial leaf linked with it. This adds a half twist to the framing of the adjacent edge of $G$, while preserving the isotopy class of $O$ and $G$. Thus $\left[S^{3}, O, G\right] \underset{k}{=}-\left[S^{3}, O, G\right]$ by Lemma 5.9 .

We are in a position to prove the second inclusion theorem.

Theorem 5.11 For each integer $n$ we have $\mathcal{F}_{3 n}^{a s} \subset \mathcal{F}_{2 n}^{Y} \otimes \mathbb{Z}[1 / 2]$.

Proof Let $O$ be a lacing pair in $S^{3}$ with $O$ having at least $n$ components. In view of Corollary 5.7, it suffices to prove that $\left[S^{3}, O, G\right] \in \mathcal{F}_{2 n}^{Y} \otimes \mathbb{Z}[1 / 2]$. We proceed by downward induction on the degree $d$ of $G$. If $d \geq 2 n$, then obviously $\left[S^{3}, O, G\right] \in \mathcal{F}_{2 n}^{Y}$ and the theorem follows. Suppose now that the inclusion holds for all Y-links of degree higher than $d$ and let us prove it for a Y-link $G$ of degree $d$.

By a repeated use of Corollary 5.8 we can reduce the problem to the case when none of the edges of $G$ pass through the discs $D_{i}$ bounding the components of $O$.

If for some $i$ the disc $D_{i}$ does not intersect $G$, then $\left[S^{3}, O, G\right]=0 \in \mathcal{F}_{2 n}^{Y}$ by Lemma 2.2, and we are done. 
If some disc $D_{i}$ intersects $G$ in exactly one point belonging to a leaf $l$ of $G$, the statement follows from Lemma 5.10 (applicable by the induction assumption).

We are left with the case when each component of $O$ is linked with at least two leaves of $G$. But each leaf of $G$ can be linked with at most one component of $O$. Therefore, $G$ should have at least $6 n$ leaves, ie, at least $2 n$ components. Hence $\left[S^{3}, O, G\right] \in \mathcal{F}_{2 n}^{Y}$, and the theorem follows.

Remark 5.12 Over the integers, a simplified version of the above counting argument leads to an inclusion $\left[S^{3}, O, G\right] \in \mathcal{F}_{n}^{Y}$ that does not require Lemmas 5.9 and 5.10. Indeed, we proceed as in the proof of Theorem 5.11 above, first reducing the problem to the case when no edges of $G$ pass through the discs $D_{i}$, and then noticing that if some $D_{i}$ does not intersect $G$, then $\left[S^{3}, O, G\right]=0$ by Lemma 2.2. Otherwise, each of the $3 n$ discs $D_{i}$ intersects at least one leaf of $G$. Each leaf of $G$ can intersect at most one disc, so $G$ should have at least $3 n$ leaves, ie, at least $n$ components, hence $\left[S^{3}, O, G\right] \in \mathcal{F}_{n}^{Y}$.

\section{The equivalence of the $\mathcal{F}^{b}$ and $\mathcal{F}^{Y}$ filtrations}

\subsection{Plan of the proof}

The section is arranged in the following way.

In the first part we shall prove an inclusion $\mathcal{F}_{2 n}^{Y} \subset \mathcal{F}_{n}^{b}$. This is done in two steps. By Corollary 5.4 the space $\mathcal{F}_{d}^{Y}$ is generated by all $\left[S^{3}, O, T\right]$, where $T$ is a trivial $d$-component $\mathrm{Y}$-link in $S^{3}$, and the link $O$ laces $T$. In the first step, we take $d=2 n^{2}$, construct a "good" sublink $B \subset O$ with at least $n$ components, and show that this implies $\mathcal{F}_{2 n^{2}}^{Y} \subset \mathcal{F}_{n}^{b}$. Building on this result, in the second step we prove a stronger inclusion $\mathcal{F}_{2 n}^{Y} \subset \mathcal{F}_{n}^{b}$ using the IHX relation.

In the second part we shall prove an opposite inclusion $\mathcal{F}_{n}^{b} \subset \mathcal{F}_{2 n}^{Y} \otimes \mathbb{Z}[1 / 2]$. We present any $n$-component boundary link as an image of a trivial unimodular link $O$ under surgery on a $\mathrm{Y}$-link $G$, linking $O$ in a special way, which we will call "1-lacing". Using this presentation together with an appropriately modified version of Lemma 5.10, we prove that $\mathcal{F}_{n}^{b} \subset \mathcal{F}_{2 n}^{Y} \otimes \mathbb{Z}[1 / 2]$ by an easy counting argument.

\subsection{Constructing a boundary sublink}

Let $T$ be a trivial $2 n^{2}$ component $\mathrm{Y}$-link in $M$ and let $O$ be a link which laces $T$. Below we construct a sublink $B \subset O$ with at least $n$ components, which 
will be a boundary link in the manifold, obtained from $M \backslash(O \backslash B)$ by surgery on $T$. This construction is based on a notion of a good sublink.

A link $B$ in $M$ is good, if $B$ laces $T$ and the following conditions hold:

- No Y-graph of $T$ is linked with $B$ by all three leaves.

- If some Y-graph of $T$ is linked with $B$ by two leaves, then it is linked with just one component of $B$.

The possible structure of $B$ in a neighborhood of a component of $T$ is shown in Figure 18.
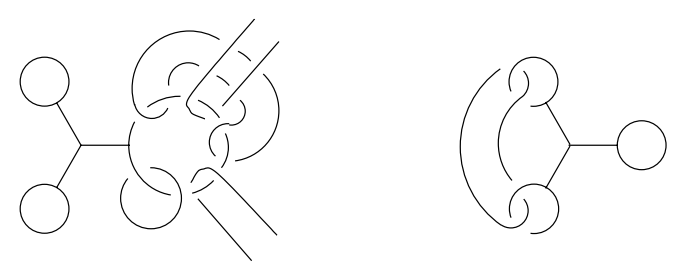

Figure 18: The structure of a good link near a Y-graph

Lemma 6.1 Let $T$ be a trivial $Y$-link in $M$ and $B$ be a good sublink. Then the image $B^{\prime}$ of $B$ in $M_{T}$ is a boundary link in a neighborhood of $T \cup D$, where $D$ is a union of discs bounding the components of $B$.

Proof Cut out a tubular neighborhood $N$ of $T$; this is a disjoint union of genus 3 handlebodies. Components of $B$ bound non-intersecting discs in $M$, which intersect the boundary $\partial N$ by either one or two circles, which are meridians of $N$. Perform surgery on $T$ by cutting, twisting by an appropriate element of the mapping class group, and gluing back the handlebodies $N$. This transforms the meridians into some other curves on $\partial N$. It suffices to show that for each handlebody $N_{i}$ of $N$ these curves on $\partial N_{i}$ are bounding inside $N_{i}$.

Let $m$ be a meridian on $\partial N_{i}$. The surgery on this $\mathrm{Y}$-graph corresponds to the action of an element of the Torelli subgroup (which acts trivially on $H_{1}\left(N_{i}\right)$ ), so the image of $m$ still bounds a surface $\Sigma_{m}$. Now, recall that $B$ is a good link, so there are only two possible configurations of the meridians. Firstly, $\partial N_{i}$ may contain just two meridians $m$ and $m^{\prime}$, corresponding to the same component $b$ of $B$. Then, smoothing the intersections of $\Sigma_{m}$ with $\Sigma_{m^{\prime}}$, we obtain a surface bounding $m \cup m^{\prime}$. Secondly, these meridians may appear only on one of the three handles of $\partial N_{i}$. Pick one of the meridians $m$; all other meridians on $\partial N_{i}$ may be considered as small push-offs of $m$ in the normal direction. Thus their 
images under the Y-surgery, together with the corresponding surfaces, can be obtained by a similar push-offs. This finishes the proof of the lemma.

Corollary 6.2 Let $T$ be a trivail $Y$-link in $M$ and $B$ be an $n$-component good link with the union $D$ of discs bounding $B$. Then $[M, L \cup B, T] \in \mathcal{F}_{n}^{b}$ for any link $L$ in $M \backslash(T \cup D)$.

Proof By Lemma 6.1, the image of B under surgery on $T^{\prime} \subset T$ bounds in the complement of $L$. Thus it will bound also in $\left(M_{T^{\prime}}\right)_{L^{\prime}}$, for any $L^{\prime} \subset L$.

Lemma 6.3 Let $T$ be a trivial $2 n^{2}$-component $Y$-link in $M$ and let $O$ be a link, which laces $T$ and links all leaves of $T$. Then there exists a good sublink $B$ of $O$ with at least $n$ components.

We will say that two components of $O$ (respectively, two leaves of $T$ ) are neighboring, if there is a $\mathrm{Y}$-graph (respectively, a component of $O$ ) which is linked with both of them.

Proof Suppose that there is a leaf of $T$, which has at least $n$ neighbors, apart from the other leaves of the same $\mathrm{Y}$-graph. Then the corresponding components of $O$ comprise $B$. It remains to consider the case when each leaf of $T$ has less than $n$ neighbors belonging to other $\mathrm{Y}$-graphs. Pick an arbitrary component $B_{1}$ of $O$ and remove from $O$ all neighboring components of $B_{1}$. Repeat this step, each time picking a new component $B_{i}$ of the remaining link, until there are no more components left. Finally, take $B=\cup_{i} B_{i}$.

Let us establish a lower bound for the number of these steps. Each $B_{i}$ is linked with at most two Y-graphs, leaves of which have altogether at most $6(n-1)-2=6 n-8$ other neighboring leaves. Thus the removal of $B_{i}$ and of the link components neighboring $B_{i}$ may unlink at most $6 n-2$ leaves of $T$. In the beginning, $O$ was linked with all $6 n^{2}$ leaves of $G$. Therefore, the number of steps is at least $\frac{6 n^{2}}{6 n-2}>n$, thus $B$ has more than $n$ components.

Remark 6.4 In fact, one can assume that the degree of $T$ is just $6 n$, but the construction of $B$ in this case is significantly more complicated.

\subsection{The inclusion $\mathcal{F}_{2 n}^{Y} \subset \mathcal{F}_{n}^{b}$}

Let us start with a weaker inclusion: 
Proposition 6.5 For each integer $n$, we have $\mathcal{F}_{2 n^{2}}^{Y} \subset \mathcal{F}_{n}^{b}$.

Proof Let $T$ be a trivial $2 n^{2}$-component $\mathrm{Y}$-link in $S^{3}$ and $O$ be an arbitrary link lacing $T$. In view of Corollary 5.4, it suffices to prove that $\left[S^{3}, O, T\right] \in \mathcal{F}_{n}^{b}$. If some leaf of $T$ is not linked with $O$, then $\left[S^{3}, O, T\right]=0 \in \mathcal{F}_{n}^{b}$. Otherwise, all leaves of $T$ are linked with $O$ and we can use Lemma 6.3 to find a good sublink $B$ of $O$ with at least $n$ components. It remains to apply Lemma 6.1 for $M=S^{3}$ and $L=O \backslash B$.

Now we are in a position to prove a stronger result:

Theorem 6.6 For each integer $n$, we have $\mathcal{F}_{2 n}^{Y} \subset \mathcal{F}_{n}^{b}$.

Proof Let $G$ be a clover of degree $d \geq 2 n$ in $S^{3}$. We proceed by the downward induction on $d$. If $d \geq 2 n^{2}$, then $\left[S^{3}, G\right] \in \mathcal{F}_{n}^{b}$ by Proposition 6.5 , and the theorem follows. Suppose that the statement holds for any clover of degree higher than $d$, and let us prove it for a clover $G$ of degree $d$. Note that by the induction assumption we can use Theorem 4.13. Thus it suffices to prove the statement for a clover $G$ which is a disjoint union of a degree $2 k \leq d$ clover $G^{\prime}$ with no leaves and $d-2 k$ copies $G_{i}$ of a Y-graph with three special leaves.

We call a path in a connected graph maximal, if it is connected, passes along each edge at most once, and contains the maximal number of edges. A path in $G^{\prime}$ is maximal, if it is maximal in each of its connected components. The number $v$ of vertices of $G^{\prime}$ which do not belong to a maximal path is called the length-defect of $G^{\prime}$. If $v$ is positive, pick a vertex of $G^{\prime}$ which does not belong to a maximal path, but is connected to it by an edge. Applying to this edge the IHX relation of Theorem 4.11, we obtain two clovers, each of which has the length defect $v-1$. Hence it suffices to prove the theorem for $v=0$. Notice that if $v=0$, there are at least $k$ edges of $G^{\prime}$ without common ends. Thus, including $G_{i}$ 's, there are at least $d-k$ edges of $G$ without common ends.

Cut all edges of $G^{\prime}$ by Theorem 2.4. Now, unlink all pairs of newly created leaves and change the framing of all leaves of $G_{i}$ 's to 0 by $K_{4}$, as shown below:

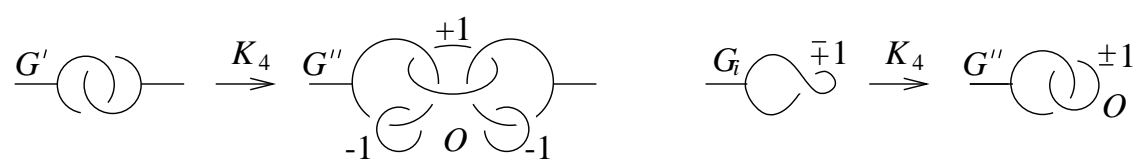

Denote by $O$ the resulting link and by $T$ the $d$-component $\mathrm{Y}$-link obtained from $T$. Clearly the link $O$ laces $T$ and

$$
\left[S^{3}, G\right]= \pm\left[S_{O}^{3}, T\right]= \pm \sum_{O^{\prime} \subset O}(-1)^{\left|O^{\prime}\right|}\left[S^{3}, O^{\prime}, T\right] .
$$


We should show that $\left[S^{3}, O^{\prime}, T\right] \in \mathcal{F}_{n}^{b}$ for each $O^{\prime} \subset O$. If some leaf of $T$ is unlinked with $O^{\prime}$, then this leaf is trivial and $\left[S^{3}, O^{\prime}, T\right]=0$. Otherwise, by the construction of $O$, there are at least $d-k \geq d / 2 \geq n$ non-neighboring components of $O^{\prime}$ (since there were at least $d-k$ edges of $G$ without common ends). These components comprise a good sublink of $O^{\prime}$. The theorem now follows from Corollary 6.2.

\subsection{Undoing a boundary link}

Let $B$ be a boundary link in $M$, and fix a surface $\Sigma=\cup_{i} \Sigma_{i}$ bounded by $B$. We would like to present $B$ by a trivial unimodular link bounding a collection of discs, together with a Y-link, lacing it in a rather special way. Namely, we will say that a $\mathrm{Y}$-link $b$-laces the link $B$, and each of its components intersects $\Sigma$ in at most one point, which belongs to a trivial leaf. By the classification of surfaces, we can assume that a surface $\Sigma_{i}$ bounding each component is an embedding of a connected sum of discs with two attached bands. Lemma 5.1 shows how such a disc with two (possibly linked and knotted) bands can be obtained from a standard disc by surgery on a $\mathrm{Y}$-graph $b$-lacing it:

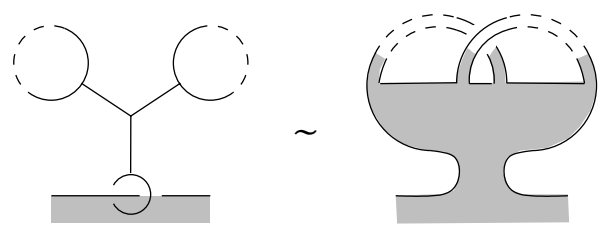

A pair $(O, G)$ consisting of a trivial link $O$ and a $\mathrm{Y}$-link $G$ in $M$ is called a $b$-lacing pair, if $G$ b-laces $O$ for $\Sigma_{i}=D_{i}$ being discs bounding the components of $O$. Exchanging pairs of bands to $b$-lacing $\mathrm{Y}$-graphs (or vice versa) as above, we obtain

Corollary 6.7 For any $n$-component boundary link $B$ in a manifold $M$ there exists a $b$-lacing pair $(O, G)$ such that surgery on $G$ transforms $O$ into $B$. Conversly, for any $b$-lacing pair $(O, G)$, surgery on $G$ transforms $O$ into a boundary link.

We illustrate this construction on an example of a genus 2 surface bounding a trefoil:

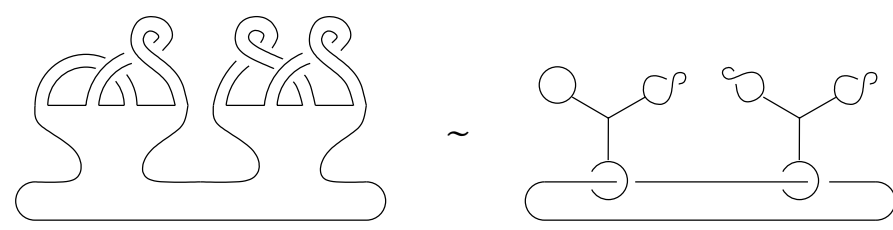


Remark 6.8 A presentation of a boundary link by a $b$-lacing pair $(O, G)$ encodes an information about the Seifert surface $\Sigma$ of $B$ and the Seifert form. Indeed, each Y-graph $G_{i}$ of $G$ corresponds to a pair of bands, ie, to a handle $h_{i}$ of $\Sigma$. The cores $b_{2 i-1}, b_{2 i}$ of these bands, being appropriately oriented and closed in the disc in a standard way, comprise a preferred basis of $H_{1}(\Sigma)$. This observation allows one to deduce that the Seifert matrix is given by $1 k\left(b_{i}, b_{j}\right)+$ $\delta_{i+1, j}$, where $1 k\left(b_{i}, b_{j}\right)$ is the linking matrix of $2 n$ non-trivial leaves of $G$. We will investigate some applications of this construction in a future paper.

Corollary 6.9 The space $\mathcal{F}_{n}^{b}$ is generated by all $\left[S^{3}, O, G\right]$, where $(O, G)$ is a $b$-lacing pair in $S^{3}$ and $O$ has at least $n$ components.

Proof By Corollary 6.7, $\mathcal{F}_{n}^{b}$ is generated by all $\left[S_{G}^{3}, O\right]$, with $G$ and $O$ as above. It remains to notice that $S_{G}^{3}=\sum_{G^{\prime} \subset G}(-1)^{\left|G^{\prime}\right|}\left[S^{3}, G^{\prime}\right]$, and that any Y-sublink $G^{\prime}$ of $G$ also $b$-laces $O$.

\subsection{The inclusion $\mathcal{F}_{n}^{b} \subset \mathcal{F}_{2 n}^{Y}$}

Returning to the proof of Lemma 5.10 (and Lemmas 5.9, 4.4 used in it), we notice that it may be restated for $b$-lacing pairs in $M$. Thus the following modified version of Lemma 5.10 holds:

Lemma 6.10 Let $(O, G)$ be a $b$-lacing pair in a manifold $M$ with $G$ of degree $d$. Suppose that some disc $D_{i}$ bounding a component of $O$ intersects $G$ in just one point. Suppose also that for any $b$-lacing pair $(O, \Gamma)$ with $\Gamma$ of degree greater than $d$ we have $[M, O, \Gamma] \underset{k}{=} 0$. Then $2[M, G] \underset{k}{=} 0$.

We are ready to prove the last inclusion theorem.

Theorem 6.11 For each integer $n$ we have $\mathcal{F}_{n}^{b} \subset \mathcal{F}_{2 n}^{Y} \otimes \mathbb{Z}[1 / 2]$.

Proof Let $(O, G)$ be a $b$-lacing pair in $S^{3}$ such that $O$ has at least $n$ components. We will show by downward induction on the degree $d$ of $G$ that $\left[S^{3}, O, G\right] \in \mathcal{F}_{2 n}^{Y} \otimes \mathbb{Z}[1 / 2]$. If $d \geq 2 n$, then obviously $\left[S^{3}, O, G\right] \in \mathcal{F}_{2 n}^{Y}$. Inductively suppose that the statement holds for any $b$-lacing pair $(O, \Gamma)$ in $S^{3}$ with $\Gamma$ of degree greater than $d$.

If for some $i$ the disc $D_{i}$ bounding a component of $O$ does not intersect $G$, then obviously $\left[S^{3}, O, G\right]=0 \in \mathcal{F}_{2 n}^{Y}$ and we are done. If for some $i$ the disc 
$D_{i}$ intersects $G$ in exactly one point, the statement follows from Lemma 6.10 (applicable by the induction assumption). We are left with the case when each disc $D_{i}$ intersects $G$ in at least two points. Since $G b$-laces $O$, these points should belong to the leaves of different $\mathrm{Y}$-graphs, which do not intersect any other disc $D_{j}, j \neq i$. Therefore, $G$ should have at least $2 n$ components, and the theorem follows.

Remark 6.12 The inclusion of Theorem 6.11 and its proof are valid not just for $\mathbb{Z}$ HS, but for arbitrary 3 -manifolds.

\section{References}

[1] T D Cochran, P M Melvin, Finite type invariants of 3-manifolds, Rice University and Bryn Mawr College preprint, November 1997, available from arxiv:math.GT/9805026

[2] S Garoufalidis, On finite type 3-manifold invariants I, J. Knot Theory and its Ramifications, 5 (1996) 441-462

[3] S Garoufalidis, J Levine, Finite type 3-manifold invariants, the mapping class group and blinks, J. Diff. Geom. 47 (1997) 257-320

[4] M Goussarov, Knotted graphs and a geometrical technique of n-equivalence, St. Petersbourg Math. Journal, to appear, POMI preprint 1995 in Russian, available at http://www.ma.huji.ac.il/ drorbn/Goussarov,

[5] M Goussarov, Finite type invariants and n-equivalence of 3-manifolds, Comp. Rend. Ac. Sci. Paris, 329 Série I (1999) 517-522

[6] K Habiro, Claspers and finite type invariants of links, Geometry and Topology, 4 (2000) 1-83

[7] D Johnson, An abelian quotient of the mapping class group, Math. Ann. 249 (1980) 225-242

[8] D Johnson, A survey of the Torelli group, from "Low-dimensional topology (San Francisco, Calif. 1981)", Contemporary Math. 20 (1983) 163-179

[9] R Kirby, A calculus for framed links in $S^{3}$, Invent. Math. 45 (1978) 35-56

[10] S Matveev, Generalized surgeries of three-dimensional manifolds and representations of homology spheres, Mat. Zametki, 42 (1987) no. 2, 268-278; English translation in Math. Notices Acad. Sci. USSR, 42:2

[11] S Matveev, M Polyak, A geometrical presentation of the surface mapping class group and surgery, Comm. Math. Phys. 160 (1994) 537-556

[12] H Murakami, Y Nakanishi, On a certain move generating link-homology, Math. Ann. 284 (1989) 75-89

[13] T Ohtsuki, Finite type invariants of integral homology 3-spheres, J. Knot Theory and its Ramifications, 5 (1996) 101-115 